\title{
Quantum SO(3) groups and quantum group actions on $M_{2}$
}

\author{
Piotr M. Sołtan*
}

\begin{abstract}
Answering a question of Shuzhou Wang we give a description of quantum $\mathrm{SO}(3)$ groups of Podleś as universal compact quantum groups acting on the $\mathrm{C}^{*}$-algebra $M_{2}$ and preserving the Powers state. We use this result to give a complete classification of all continuous compact quantum group actions on $\mathrm{M}_{2}$.
\end{abstract}

Mathematics Subject Classification (2010). 46L89, 58B34, 58B32; 17B37, $16 \mathrm{~W} 30$.

Keywords. Quantum group, quantum group action, quantum family of maps.

\section{Introduction}

The classical group $\mathrm{SO}(3)$ has many useful descriptions. For example $\mathrm{SO}(3)$ is the automorphism group of the $\mathrm{C}^{*}$-algebra $M_{2}$ of $2 \times 2$ complex matrices. In this paper we will focus on quantum $\mathrm{SO}(3)$ groups first defined by Piotr Podleś in [8].

In his paper [16] Shuzhou Wang asked if the quantum SU(2) group could be described as a quantum automorphism group of $M_{2}$ endowed with a collection of functionals ([16], Remark (4) on p. 209). We solve this problem after a necessary modification. Namely the quantum SU(2) must be replaced by the quantum $\mathrm{SO}(3)$ group (the confusion stems from an erroneous identification in [16] of the classical $\mathrm{SU}(2)$ as the automorphism group of $M_{2}$ ).

We will show that the quantum groups $\mathrm{S}_{q} \mathrm{O}(3)$ of Podleś are the universal compact quantum groups acting continuously on $M_{2}$ and preserving the Powers state

$$
\omega_{q}: M_{2} \ni\left[\begin{array}{ll}
m_{1,1} & m_{2,2} \\
m_{2,1} & m_{2,2}
\end{array}\right] \longmapsto \frac{1}{1+q^{2}}\left(m_{1,1}+q^{2} m_{2,2}\right),
$$

where $q$ is any fixed number in $] 0,1]$. In other words $\mathrm{S}_{q} \mathrm{O}(3)$ is the quantum automorphism group of the quantum space underlying $M_{2}$ preserving the state $\omega_{q}$. More details will be given in subsequent sections.

The quantum $\mathrm{SO}(3)$ groups have originally been defined either as quotient groups of quantum SU(2) or by means of a complicated system of generators and relations

\footnotetext{
* Research partially supported by Polish government grants no. 115/E-343/SPB/6.PRUE/DIE50/20052008 and N201 177033.
} 
(see Section 3). Therefore our description of these groups as universal objects in the category of compact quantum groups acting continuously on $M_{2}$ and preserving the Powers state yields a possible alternative approach to these quantum groups which avoids some technical complexity.

In a recent development a different and exciting description of quantum $\mathrm{SO}(3)$ groups has been found. In [5] D. Goswami introduced the notion of a quantum isometry group of a noncommutative manifold. A number of examples of these quantum groups were found in [5] and [2]. The famous quantum spheres of Podles ([8]) have been given a structure of a noncommutative manifold in [4], and it was proved by J. Bhowmick and D. Goswami in [1] that the quantum isometry groups of these noncommutative manifolds are precisely the quantum $\mathrm{SO}(3)$ groups. From this point of view the quantum $\mathrm{SO}(3)$ groups are described as universal objects acting on the quantum manifolds $S_{c, q}^{2}$. This description is closely related to our approach. Moreover the notion of a quantum family of maps (see Section 1.1.2) also appears in the approach of J. Bhowmick and D. Goswami.

Finally let us note that the new description of Podleś quantum $\mathrm{SO}(3)$ groups gives, together with some other results, a complete description of continuous compact quantum group actions on two by two complex matrices (see Section 1.2 and Section 6).

\subsection{Terminology}

1.1.1. Quantum spaces. We will use the language of quantum groups and quantum spaces (cf. [17], [18], [8], [10], [14]). A quantum space is, by definition, an object of the category dual to the category of $C^{*}$-algebras, as defined in [20], Section 0 (see also [17]). In this category a morphism from a $C^{*}$-algebra $\mathfrak{U}$ to a $C^{*}$-algebra $\mathfrak{C}$ is a non-degenerate *-homomorphism from $\mathfrak{U}$ to $\mathrm{M}(\mathfrak{C})$, where $\mathrm{M}(\mathfrak{C})$ is the multiplier algebra of $\mathfrak{C}$. However, in this paper all $\mathrm{C}^{*}$-algebras will be unital (in other words all quantum spaces will be compact) which implies that morphisms will simply be unital *-homomorphisms. We will write $\operatorname{Mor}(\mathfrak{U}, \mathfrak{C})$ for the set of all morphisms from $\mathfrak{U}$ to $\mathfrak{C}$. For a given $\mathrm{C}^{*}$-algebra $\mathfrak{C}$ we will write $\mathcal{L} \mathcal{S}(\mathfrak{C})$ for the corresponding quantum space. Let us stress that by introducing the notion of a quantum space we are not defining a new concept but simply create a language in which it seems easier to express some mathematical ideas. Following Piotr Podleś ([8], [10]) we will sometimes use the notation $\mathrm{C}(\mathfrak{X})$, where $\mathfrak{X}$ is some quantum space. Examples of this are the well-known $\mathrm{C}^{*}$-algebras $\mathrm{C}\left(\mathrm{S}_{q} \mathrm{O}(3)\right)$ and $\mathrm{C}\left(\mathrm{S}_{q} \mathrm{U}(2)\right)$ of [8], [10] and [18].

1.1.2. Quantum families of maps, quantum semigroups and their actions. We consider compact quantum groups and semigroups and their actions on quantum spaces. These are special cases of quantum families of maps defined and studied in [14]. To explain this notion let us consider three quantum spaces $\mathcal{L}(N), \mathcal{Q S}(M)$ and $Q \mathcal{S}(C)$ (in other words let $N, M$ and $C$ be $C^{*}$-algebras). A quantum family of maps $\mathcal{L S}(M) \rightarrow \mathcal{Q S}(N)$ labeled by $\mathcal{Q S}(C)$ is a morphism $\Psi \in \operatorname{Mor}(N, M \otimes C)$. 
This notion was introduced already in [17] and generalizes the classical notion of a continuous family of continuous maps of locally compact spaces. In case $M$ is finite dimensional and $N$ is finitely generated and unital there exists a special quantum space $Q \mathcal{S}(\mathfrak{C})$ and a quantum family $\Phi \in \operatorname{Mor}(N, M \otimes \mathfrak{S})$ called the quantum family of all maps $Q \mathcal{S}(M) \rightarrow \mathcal{L S}(N)$. It is distinguished by the property that for any $\mathrm{C}^{*}$ algebra $D$ and any $\Psi_{D} \in \operatorname{Mor}(N, M \otimes D)$ there exists a unique $\Lambda \in \operatorname{Mor}(\mathfrak{C}, D)$ such that the diagram

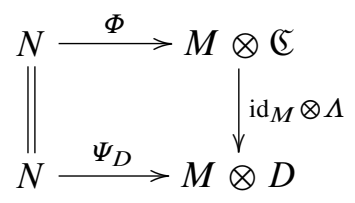

is commutative. The quantum space $\mathcal{Q S}(\mathfrak{S})$ labeling $\Phi$ is called the quantum space of all maps $Q \mathcal{S}(M) \rightarrow \mathcal{L S}(N)$. In [14] we show that for $N=M$ the quantum space of all maps $\mathcal{L S}(M) \rightarrow \mathcal{L S}(M)$ is a compact quantum semigroup with unit. In other words there exists a comultiplication $\Delta_{\mathfrak{C}} \in \operatorname{Mor}(\mathfrak{C}, \mathfrak{C} \otimes \mathfrak{C})$ and a counit $\epsilon_{\mathfrak{C}} \in \operatorname{Mor}(\mathfrak{C}, \mathbb{C})$. Moreover $\Phi$ is then an action of $\left(\mathfrak{C}, \Delta_{\mathfrak{C}}\right)$ on $M$ :

$\left(\Phi \otimes \operatorname{id}_{\mathfrak{C}}\right) \circ \Phi=\left(\operatorname{id}_{M} \otimes \Delta_{\mathfrak{C}}\right) \circ \Phi$.

As in [14], the quantum semigroup of all maps $\mathcal{L S}(M) \rightarrow \mathcal{L S}(M)$ will be denoted by the symbol $\mathcal{Q}-\operatorname{Map}(\mathcal{Q S}(M))$.

In [14] we also studied some subsemigroups of $\mathcal{Q}-\operatorname{Map}(\mathcal{L S}(M))$. Those that are important for this paper are the semigroups preserving distinguished states on $M$. Let $\omega$ be a state on $M$ and let $\Psi \in \operatorname{Mor}(M, M \otimes D)$ be a quantum family of maps. We say that $\Psi$ preserves $\omega$ if

$$
\left(\omega \otimes \operatorname{id}_{D}\right) \Psi(m)=\omega(m) \mathbb{1}
$$

for all $m \in M$. By [14], Theorem 5.4, there exist a special quantum space $Q \mathcal{S}(\boldsymbol{C})$ and a quantum family $\boldsymbol{\Phi} \in \operatorname{Mor}(M, M \otimes C)$ with the property that for any quantum family $\Psi_{D} \in \operatorname{Mor}(M, M \otimes D)$ preserving $\omega$ there exists a unique $\Lambda \in \operatorname{Mor}(C, D)$ such that

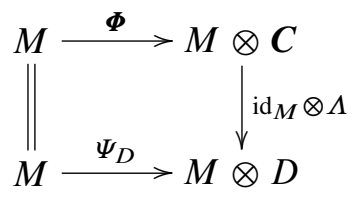

is commutative. The quantum space $\mathcal{L}(\boldsymbol{C})$ is naturally endowed with the structure of a quantum semigroup with unit, which we denote by $2-\operatorname{Map}^{\omega}(\mathcal{Q S}(M))$. Moreover $\boldsymbol{\Phi}$ is an action of $\left.\mathcal{Q}-\operatorname{Map}^{\omega} \mathcal{Q S}(M)\right)$ on $\mathcal{Q S}(M)$.

Let $\left(\mathfrak{B}, \Delta_{\mathfrak{B}}\right)$ and $\left(\mathfrak{C}, \Delta_{\mathfrak{C}}\right)$ be two quantum semigroups. A morphism $\Gamma \in$ $\operatorname{Mor}(\mathfrak{B}, \mathfrak{C})$ is called a quantum semigroup morphism if

$$
(\Gamma \otimes \Gamma) \circ \Delta_{\mathfrak{B}}=\Delta_{\mathfrak{C}} \circ \Gamma
$$


In case $\left(\mathfrak{B}, \Delta_{\mathfrak{B}}\right)$ and $\left(\mathfrak{C}, \Delta_{\mathfrak{C}}\right)$ are compact quantum groups ([21], Definition 2.1$)$ a morphism $\Gamma$ satisfying (2) is called a quantum group morphism.

1.1.3. Actions of compact quantum groups and the Podleś condition. In this paper we will mainly consider actions of compact quantum groups on quantum spaces. Let $\mathcal{E}=\left(\mathfrak{B}, \Delta_{\mathfrak{B}}\right)$ be a compact quantum group and let $M$ be a $\mathrm{C}^{*}$-algebra. As in the case of quantum semigroup actions we say that $\Psi_{\mathfrak{B}} \in \operatorname{Mor}(M, M \otimes \mathfrak{B})$ is an action of $\mathcal{E}$ on $Q \mathcal{S}(M)$ if

$$
\left(\Psi_{\mathfrak{B}} \otimes \operatorname{id}_{\mathfrak{B}}\right) \circ \Psi_{\mathfrak{B}}=\left(\operatorname{id}_{M} \otimes \Delta_{\mathfrak{B}}\right) \circ \Psi_{\mathfrak{B}}
$$

We say that $\Psi_{\mathfrak{B}}$ satisfies Podleś condition if the set

$$
\left\{\Psi_{\mathfrak{B}}(m)(\mathbb{1} \otimes b) \mid m \in M, b \in \mathfrak{B}\right\}
$$

is linearly dense in $M \otimes \mathfrak{B}$. This condition was formulated by Piotr Podleś in [9], Definicja 2.2. It appeared in many later publications by various authors (e.g. [3], Definition 1, [7], Definition 3.1, [15], Definition 2.6). An even stronger definition of an action was used in [16], Definition 2.1. Following [15] we will call actions of compact quantum groups satisfying the Podleś condition continuous actions. This condition is always satisfied by actions of classical groups.

An important class of compact quantum group actions are the ergodic actions. Since actions are particular cases of quantum families of maps, let us define the notion of an ergodic quantum family. Let $M$ be a $C^{*}$-algebra and let $\Psi_{D} \in \operatorname{Mor}(M, M \otimes D)$ be a quantum family of maps $Q \mathcal{S}(M) \rightarrow Q \mathcal{S}(M)$ labeled by a quantum space $Q \mathcal{S}(D)$. We say that $\Psi_{D}$ is ergodic if for all $m \in M$ the condition that $\Psi_{D}(m)=m \otimes \mathbb{1}$ implies

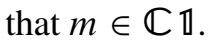

1.2. Statement of main results. For $q \in[0,1]$ let $\omega_{q}$ be the state on $M_{2}$ introduced by (1). When $q>0$, the quantum group $\mathrm{S}_{q} \mathrm{O}(3)$ acts continuously on $\mathcal{Q S}\left(M_{2}\right)$ preserving $\omega_{q}$. We denote the morphism describing this action by $\Psi_{q}$. The core result of this paper can be stated in the following way:

Theorem 1.1. Fix $q \in] 0,1]$. Let $\mathscr{E}=\left(\mathfrak{B}, \Delta_{\mathfrak{B}}\right)$ be a compact quantum group and let $\Psi_{\mathfrak{B}} \in \operatorname{Mor}\left(M_{2}, M_{2} \otimes \mathfrak{B}\right)$ be a continuous action of $\mathcal{G}$ on $M_{2}$ preserving the state $\omega_{q}$. Then there exists a unique $\Gamma \in \operatorname{Mor}\left(\mathrm{C}\left(\mathrm{S}_{q} \mathrm{O}(3)\right), \mathfrak{B}\right)$ such that

$$
\left(\mathrm{id}_{M_{2}} \otimes \Gamma\right) \circ \Psi_{q}=\Psi_{\mathfrak{B}} .
$$

Moreover $\Gamma$ is a quantum group morphism.

In other words the quantum group $\mathrm{S}_{q} \mathrm{O}(3)$ is the universal quantum group acting continuously on $M_{2}$ and preserving the Powers state $\omega_{q}$.

In Section 5.2 we treat the case of $q=0$ and show that the universal quantum group acting continuously on $\mathcal{Q S}\left(M_{2}\right)$ and preserving $\omega_{0}$ is the classical group $\mathbb{T}$. 
With these results we give a complete description of all continuous compact quantum group actions on $\mathcal{Q S}\left(M_{2}\right)$ in Section 6. A special uniqueness result is given for ergodic actions (cf. Theorem 6.1).

\subsection{A tool from operator theory}

Theorem 1.2 (Fuglede-Putnam-Rosenbloom). Let $\mathfrak{C}$ be a $C^{*}$-algebra and let $n_{1}, n_{2}$, $a \in \mathfrak{C}$. Assume that $n_{1}$ and $n_{2}$ are normal and that a $n_{1}=n_{2} a$. Then $a n_{1}^{*}=n_{2}^{*} a$.

The proof of Theorem 1.2 can be found in [12], Section 12.16. We will use this theorem only in the very special case when $n_{2}=\lambda n_{1}$ for some fixed $\lambda \in \mathbb{R}$. Therefore, if $n$ is a normal element of a $C^{*}$-algebra $\mathfrak{C}$ and

$$
a n=\lambda n a
$$

for some $a \in \mathfrak{C}$, then

$$
a n^{*}=\lambda n^{*} a
$$

\section{Quantum semigroups preserving states on $M_{2}$}

2.1. Quantum semigroup of all maps $\mathcal{Q S}\left(M_{2}\right) \rightarrow \mathcal{Q S}\left(M_{2}\right)$. The $\mathrm{C}^{*}$-algebra $M_{2}$ is generated by a single element $n$ satisfying $n^{2}=0, n n^{*}+n^{*} n=\mathbb{1}$. We take

$$
\boldsymbol{n}=\left[\begin{array}{ll}
0 & 1 \\
0 & 0
\end{array}\right] \text {. }
$$

In what follows we will define morphisms from $M_{2}$ simply by indicating the image of $\boldsymbol{n}$.

The following proposition gives a detailed description of the compact quantum semigroup Q-Map $\left(\mathcal{Q S}\left(M_{2}\right)\right)$ together with its action on $Q \mathcal{S}\left(M_{2}\right)$.

Proposition 2.1. We have $\mathcal{Q}-\operatorname{Map}\left(\mathcal{Q S}\left(M_{2}\right)\right)=(\mathbb{A}, \triangle)$, where $\mathbb{A}$ is the universal $C^{*}$-algebra generated by four elements $\alpha, \beta, \gamma$ and $\&$ satisfying the relations

$$
\begin{aligned}
& \alpha^{*} \alpha+\gamma^{*} \gamma+\alpha \alpha^{*}+\beta \beta^{*}=\mathbb{1}, \quad \alpha^{2}+\beta \gamma=0, \\
& \alpha^{*} \beta+\gamma^{*} \delta+\alpha \gamma^{*}+\beta \mathscr{\delta}^{*}=0, \quad \alpha \beta+\beta \delta=0, \\
& \beta^{*} \beta+\delta^{*} \delta+\gamma \gamma^{*}+\delta \mathscr{\delta}^{*}=1, \quad \gamma \alpha+\delta \gamma=0, \\
& \gamma \beta+\delta^{2}=0 \text {. }
\end{aligned}
$$

Moreover, the quantum semigroup structure on $\mathrm{Q}-\operatorname{Map}\left(\mathrm{QS}\left(M_{2}\right)\right)$ is given by 
$\triangle \in \operatorname{Mor}(\mathbb{A}, \mathbb{A} \otimes \mathbb{A})$, acting on the generators by

$$
\begin{aligned}
& \triangle(\alpha)=\alpha \alpha^{*} \otimes \alpha+\beta \beta^{*} \otimes \alpha+\alpha \otimes \beta+\alpha^{*} \otimes \gamma+\alpha^{*} \alpha \otimes \delta+\gamma^{*} \gamma \otimes \delta, \\
& \triangle(\beta)=\alpha \gamma^{*} \otimes \alpha+\beta \delta^{*} \otimes \alpha+\beta \otimes \beta+\gamma^{*} \otimes \gamma+\alpha^{*} \beta \otimes \delta+\gamma^{*} \delta \otimes \delta, \\
& \triangle(\gamma)=\gamma \alpha^{*} \otimes \alpha+\delta \beta^{*} \otimes \alpha+\gamma \otimes \beta+\beta^{*} \otimes \gamma+\beta^{*} \alpha \otimes \delta+\delta^{*} \gamma \otimes \delta, \\
& \triangle(\delta)=\gamma \gamma^{*} \otimes \alpha+\delta \delta^{*} \otimes \alpha+\delta \otimes \beta+\delta^{*} \otimes \gamma+\beta^{*} \beta \otimes \delta+\delta^{*} \delta \otimes \delta,
\end{aligned}
$$

while the counit $\Subset$ maps $\alpha, \gamma$ and $\&$ to 0 and $\beta$ to 1 .

The action $\Phi \in \operatorname{Mor}\left(M_{2}, M_{2} \otimes \mathbb{A}\right)$ of $\mathcal{Q}-\operatorname{Map}\left(\mathcal{Q S}\left(M_{2}\right)\right)$ on $\mathcal{Q S}\left(M_{2}\right)$ is given by

$$
\Phi(\boldsymbol{n})=\boldsymbol{n} \boldsymbol{n}^{*} \otimes \propto+\boldsymbol{n} \otimes \beta+\boldsymbol{n}^{*} \otimes \gamma+\boldsymbol{n}^{*} \boldsymbol{n} \otimes \delta=\left[\begin{array}{ll}
\alpha & \beta \\
\gamma & \delta
\end{array}\right] .
$$

The proof of Proposition 2.1 is quite straightforward and can be found in [13], Proposition 4.1.

2.2. Quantum semigroup preserving Powers states on $\boldsymbol{M}_{2}$. Choose $\left.q \in\right] 0,1[$ and let $\omega_{q}$ be the state on $M_{2}$ given by

$$
\omega_{q}\left(\left[\begin{array}{ll}
m_{1,1} & m_{1,2} \\
m_{2,1} & m_{2,2}
\end{array}\right]\right)=\frac{1}{1+q^{2}}\left(m_{1,1}+q^{2} m_{2,2}\right) .
$$

In Proposition 2.2 we give a detailed description of the quantum semigroup Q-Map ${ }^{\omega_{q}}\left(\mathcal{Q S}\left(M_{2}\right)\right)$. We shall denote the $\mathrm{C}^{*}$-algebra with comultiplication corresponding to this quantum semigroup by $(\boldsymbol{A}, \boldsymbol{\Delta})$. The action of $\mathcal{Q}-\mathrm{Map}^{\omega_{q}}\left(\mathcal{Q S}\left(M_{2}\right)\right)$ on $\mathcal{L S}\left(M_{2}\right)$ will be described by $\boldsymbol{\Phi} \in \operatorname{Mor}\left(M_{2}, M_{2} \otimes \boldsymbol{A}\right)$ and the counit of $(\boldsymbol{A}, \boldsymbol{\Delta})$ will be denoted by $\epsilon$.

Proposition 2.2. Let $\mathcal{Q}_{-M^{-}}{ }^{\omega_{q}}\left(\mathcal{Q S}\left(M_{2}\right)\right)=(\boldsymbol{A}, \boldsymbol{\Delta})$. Then $\boldsymbol{A}$ is the universal $C^{*_{-}}$ algebra generated by three elements $\beta, \gamma$ and $\delta$ satisfying

$$
\begin{aligned}
q^{4} \delta^{*} \delta+\gamma^{*} \gamma+q^{4} \delta \delta^{*}+\beta \beta^{*} & =\mathbb{1}, & & \beta \gamma=-q^{4} \delta^{2}, \\
\beta^{*} \beta+\delta^{*} \delta+\gamma \gamma^{*}+\delta \delta^{*} & =\mathbb{1}, & & \gamma \beta=-\delta^{2}, \\
\gamma^{*} \delta-q^{2} \delta^{*} \beta+\beta \delta^{*}-q^{2} \delta \gamma^{*} & =0, & & \beta \delta=q^{2} \delta \beta, \\
\delta \gamma & =q^{2} \gamma \delta & &
\end{aligned}
$$

and

$$
\begin{aligned}
& q^{4} \delta \delta^{*}+\beta \beta^{*}+q^{2} \gamma \gamma^{*}+q^{2} \delta \delta^{*}=\mathbb{1}, \\
& q^{4} \delta^{*} \delta+\gamma^{*} \gamma+q^{2} \beta^{*} \beta+q^{2} \delta^{*} \delta=q^{2} 1 \text {. }
\end{aligned}
$$


The comultiplication $\boldsymbol{\Delta} \in \operatorname{Mor}(\boldsymbol{A}, \boldsymbol{A} \otimes \boldsymbol{A})$ is

$$
\begin{aligned}
& \boldsymbol{\Delta}(\beta)=q^{4} \delta \gamma^{*} \otimes \delta-q^{2} \beta \delta^{*} \otimes \delta+\beta \otimes \beta+\gamma^{*} \otimes \gamma-q^{2} \delta^{*} \beta \otimes \delta+\gamma^{*} \delta \otimes \delta, \\
& \boldsymbol{\Delta}(\gamma)=q^{4} \gamma \delta^{*} \otimes \delta-q^{2} \delta \beta^{*} \otimes \delta+\gamma \otimes \beta+\beta^{*} \otimes \gamma-q^{2} \beta^{*} \delta \otimes \delta+\delta^{*} \gamma \otimes \delta, \\
& \boldsymbol{\Delta}(\delta)=-q^{2} \gamma^{*} \gamma \otimes \delta-q^{2} \delta \delta^{*} \otimes \delta+\delta \otimes \beta+\delta^{*} \otimes \gamma+\beta^{*} \beta \otimes \delta+\delta^{*} \delta \otimes \delta .
\end{aligned}
$$

The action of $\mathcal{Q}-\operatorname{Map}^{\omega_{q}}\left(\mathcal{Q S}\left(M_{2}\right)\right)$ on $\mathcal{Q S}\left(M_{2}\right)$ is given by $\boldsymbol{\Phi} \in \operatorname{Mor}\left(M_{2}, M_{2} \otimes \boldsymbol{A}\right)$ defined by

$$
\boldsymbol{\Phi}(\boldsymbol{n})=\left[\begin{array}{cc}
-q^{2} \delta & \beta \\
\gamma & \delta
\end{array}\right] .
$$

The counit $\epsilon$ maps $\gamma$ and $\delta$ to 0 and $\beta$ to 1 .

Proof. We know from [14], Theorem 5.4, that $\boldsymbol{A}$ is a quotient of $\mathbb{A}$ by the ideal generated by the set

$$
\left\{\left(\omega_{q} \otimes \operatorname{id}_{\mathbb{A}}\right) \Phi(m)-\omega_{q}(m) \mathbb{1} \mid m \in M_{2}\right\} .
$$

Let $\alpha, \beta, \gamma$ and $\delta$ be images of $\alpha, \beta, \gamma$ and $\delta$ under the quotient map $\pi: \mathbb{A} \rightarrow \boldsymbol{A}$. Clearly $\alpha, \beta, \gamma, \delta$ generate $\boldsymbol{A}$. We have

$$
\boldsymbol{\Phi}=\left(\mathrm{id}_{M_{2}} \otimes \pi\right) \circ \Phi
$$

so

$$
\begin{aligned}
\left(\omega_{q} \otimes \mathrm{id}_{\boldsymbol{A}}\right) \boldsymbol{\Phi}\left(\boldsymbol{n} \boldsymbol{n}^{*}\right) & =\frac{1}{1+q^{2}}\left(\alpha \alpha^{*}+\beta \beta^{*}+q^{2} \gamma \gamma^{*}+q^{2} \delta \delta^{*}\right), \\
\left(\omega_{q} \otimes \mathrm{id}_{\boldsymbol{A}}\right) \boldsymbol{\Phi}(\boldsymbol{n}) & =\frac{1}{1+q^{2}}\left(\alpha+q^{2} \delta\right), \\
\left(\omega_{q} \otimes \mathrm{id}_{\boldsymbol{A}}\right) \boldsymbol{\Phi}\left(\boldsymbol{n}^{*}\right) & =\frac{1}{1+q^{2}}\left(\alpha^{*}+q^{2} \delta^{*}\right), \\
\left(\omega_{q} \otimes \mathrm{id}_{\boldsymbol{A}}\right) \boldsymbol{\Phi}\left(\boldsymbol{n}^{*} \boldsymbol{n}\right) & =\frac{1}{1+q^{2}}\left(\alpha^{*} \alpha+\gamma^{*} \gamma+q^{2} \beta^{*} \beta+q^{2} \delta^{*} \delta\right) .
\end{aligned}
$$

On the other hand

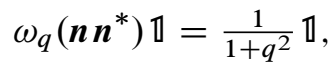

$$
\begin{aligned}
& \omega_{q}\left(n^{*} n\right) \mathbb{1}=\frac{q^{2}}{1+q^{2}} \rrbracket
\end{aligned}
$$

and $\omega_{q}(\boldsymbol{n}) \mathbb{1}=\omega_{q}\left(\boldsymbol{n}^{*}\right) \mathbb{1}=0$. It follows from [14], Theorem 5.4(1), that $\left(\omega_{q} \otimes \mathrm{id}\right) \boldsymbol{\Phi}(m)=\omega_{q}(m) \rrbracket$ for all $m \in M_{2}$. In particular we must have $\alpha=-q^{2} \delta$ and taking into account the relations (3) we see that the generators $\beta, \gamma, \delta$ must satisfy (7). Relations (8) also follow by considering (12a), (12b) and (13).

By [14], Theorem 5.4 (5), the quotient map $\pi$ is a quantum semigroup morphism, so formulas (9) follow directly form (4). Similarly we determine the values of $\boldsymbol{\epsilon}$. Finally (10) is a consequence of (5) and (11). 
So far we know that $\boldsymbol{A}$ is a $\mathrm{C}^{*}$-algebra generated by $\beta, \gamma$ and $\delta$ with relations (7) and (8). To see that it is the universal $\mathrm{C}^{*}$-algebra for these relations we use the universal property of $\mathcal{Q}-\operatorname{Map}^{\omega_{q}}\left(\mathcal{Q S}\left(M_{2}\right)\right)$. One can easily show that the universal $\mathrm{C}^{*}$-algebra $\tilde{\boldsymbol{A}}$ generated $\beta, \gamma$ and $\delta$ with relations (7) and (8) does admit a morphism $\boldsymbol{\Phi}$ as defined by (10). Moreover, since $\left\{\boldsymbol{n} \boldsymbol{n}^{*}, \boldsymbol{n}, \boldsymbol{n}^{*}, \boldsymbol{n}^{*} \boldsymbol{n}\right\}$ is a basis for $M_{2}$, we see that the quantum family of maps $\boldsymbol{\Phi} \in \operatorname{Mor}\left(M_{2}, M_{2} \otimes \tilde{\boldsymbol{A}}\right)$ preserves the state $\omega_{q}$. It follows that $\boldsymbol{A}=\tilde{\boldsymbol{A}}$.

The symbols

\section{$\boldsymbol{A}, \boldsymbol{\Delta}, \boldsymbol{\Phi}$}

will from now on be reserved exclusively to denote the objects describing the quantum semigroup structure of $\mathcal{Q}-\operatorname{Map}^{\omega_{q}}\left(\mathcal{Q S}\left(M_{2}\right)\right)$ and its action on $\mathcal{Q S}\left(M_{2}\right)$. In Section 5 we will use them also for the case of $q=1$ and $q=0$.

The universal property of $\mathcal{Q}-\operatorname{Map}^{\omega_{q}}\left(\mathcal{Q S}\left(M_{2}\right)\right)$ guarantees that for any $\mathrm{C}^{*}$-algebra $\mathfrak{B}$ and any quantum family $\Psi_{\mathfrak{B}} \in \operatorname{Mor}\left(M_{2}, M_{2} \otimes \mathfrak{B}\right)$ preserving the state $\omega_{q}$ on $M_{2}$, there exists a unique map $\Lambda \in \operatorname{Mor}(\boldsymbol{A}, \mathfrak{B})$ such that $\Psi_{\mathfrak{B}}=\left(\operatorname{id}_{M_{2}} \otimes \Lambda\right) \circ \boldsymbol{\Phi}$. By [14], Theorem 5.4(6), if $\mathfrak{B}$ admits a comultiplication $\Delta_{\mathfrak{B}}$ such that $\Psi_{\mathfrak{B}}$ satisfies $\left(\Psi_{\mathfrak{B}} \otimes \mathrm{id}_{\mathfrak{B}}\right) \circ \Psi_{\mathfrak{B}}=\left(\mathrm{id}_{M_{2}} \otimes \Delta_{\mathfrak{B}}\right) \circ \Psi_{\mathfrak{B}}$ then $\Lambda$ is a quantum semigroup morphism, (in fact one need not even demand that $\Delta_{\mathfrak{B}}$ be coassociative, cf. [14], Proposition 4.7).

\section{Podleś quantum SO(3) groups}

In [8], Remark 3, and [10], Section 3, Piotr Podleś introduced quantum groups $\mathrm{S}_{q} \mathrm{O}(3)$ as quotient groups of $\mathrm{S}_{q} \mathrm{U}(2)$ for $\left.q \in\right]-1,1[\backslash\{0\}$. In [10, Proposition 3.1] we find the following description of the $\mathrm{C}^{*}$-algebra $\mathrm{C}\left(\mathrm{S}_{q} \mathrm{O}(3)\right)$ :

Proposition 3.1 (Podleś). The $C^{*}$-algebra $\mathrm{C}\left(\mathrm{S}_{q} \mathrm{O}(3)\right)$ is the universal $C^{*}$-algebra generated by five elements $A, C, G, K, L$ satisfying

$$
\begin{aligned}
L^{*} L & =(\mathbb{1}-K)\left(\mathbb{1}-q^{-2} K\right) \\
L L^{*} & =\left(\mathbb{1}-q^{2} K\right)\left(\mathbb{1}-q^{4} K\right) \\
G^{*} G & =G G^{*} \\
K^{2} & =G^{*} G \\
A^{*} A & =K-K^{2} \\
A A^{*} & =q^{2} K-q^{4} K^{2} \\
C^{*} C & =K-K^{2} \\
C C^{*} & =q^{2} K-q^{4} K^{2} \\
L K & =q^{4} K L \\
G K & =K G
\end{aligned}
$$$$
A K=q^{2} K A,
$$$$
C K=q^{2} K C \text {, }
$$$$
L G=q^{4} G L \text {, }
$$$$
L A=q^{2} A L \text {, }
$$$$
A G=q^{2} G A \text {, }
$$$$
A C=C A \text {, }
$$$$
L G^{*}=q^{4} G^{*} L,
$$$$
A^{2}=q^{-1} L G,
$$$$
A^{*} L=q^{-1}(\mathbb{1}-K) C \text {, }
$$$$
K^{*}=K \text {. }
$$ 
Remark 3.2. (1) Using [10], Lemma 3.2, one can show that the generators $A, C, G$, $K, L$ of $\mathrm{C}\left(\mathrm{S}_{q} \mathrm{O}(3)\right)$ satisfy

$$
K=A^{*} A+G^{*} G \quad \text { and } \quad C=q^{-1} L A^{*}+q^{2} A G^{*} .
$$

Therefore one can express every relation from the list (14) using only $A, G$ and $L$.

(2) The quantum groups $\mathrm{S}_{q} \mathrm{U}(2)$ are defined for the deformation parameter $q \in]-1,1\left[\backslash\{0\}\right.$. Thus the procedure of taking a quotient by a $\mathbb{Z}_{2}$ action yields $\mathrm{S}_{q} \mathrm{O}(3)$ also for negative values of $q$. However, in [10], Proposition 3.3, Podleś shows that the quantum $\mathrm{SO}(3)$ groups defined for negative deformation parameters are isomorphic to those for positive $q$ (namely $\mathrm{S}_{-q} \mathrm{O}(3) \cong \mathrm{S}_{q} \mathrm{O}(3)$ ). Therefore it is enough to consider only positive $q$, and we will do so in what follows.

The comultiplication $\Delta_{q}$ on $\mathrm{C}\left(\mathrm{S}_{q} \mathrm{O}(3)\right)$ acts on generators in the following way:

$$
\begin{aligned}
& \Delta_{q}(A)=\left(\mathbb{1}-q^{2} K\right) \otimes A+A \otimes L-q A^{*} \otimes G-K \otimes A, \\
& \Delta_{q}(C)=-q^{2} C \otimes K+L \otimes C-q G^{*} \otimes C^{*}+C \otimes(\mathbb{1}-K), \\
& \Delta_{q}(G)=C^{*} \otimes A+G \otimes L-q^{-1} L^{*} \otimes G+q^{-2} C^{*} \otimes A \text {, } \\
& \Delta_{q}(K)=K \otimes\left(\mathbb{1}-q^{2} K\right)+q^{-1} A \otimes C+q^{-1} A^{*} \otimes C^{*}+(\mathbb{1}-K) \otimes K, \\
& \Delta_{q}(L)=-q C \otimes A+L \otimes L+q^{2} G^{*} \otimes G-q^{-1} C \otimes A \text {. }
\end{aligned}
$$

3.1. Reduction of Podleś relations. The relations (14) contain some superfluous ones. Clearly (14t) follows immediately from (14d) and (14e) as $K=A^{*} A+G^{*} G$. Moreover, since $G$ is normal (by $(14 \mathrm{c})$ ), we have $\left[G, G^{*} G\right]=0$ and, by (14o) and Theorem 1.2,

$$
A G=q^{2} G A, \quad A G^{*}=q^{2} G^{*} A,
$$

which can be rewritten as

$$
G A^{*}=q^{2} A^{*} G, \quad G A=q^{-2} A G,
$$

so that $\left[G, A^{*} A\right]=0$, which implies (14j). Finally (14q) follows from (14m) and (14c) by Theorem 1.2.

Proposition 3.3. Let $H$ be a Hilbert space and let $A, C, K \in \mathrm{B}(H)$ be such that

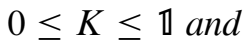

$$
\begin{aligned}
A^{*} A=C^{*} C & =K-K^{2}, \\
A A^{*}=C C^{*} & =q^{2} K-q^{4} K^{2}, \\
A K & =q^{2} K A, \\
A C & =C A .
\end{aligned}
$$

Then

$$
C K=q^{2} K C
$$


Before proving Proposition 3.3 let us introduce a (simplified version of a) very convenient notation used by S. L. Woronowicz in, for instance, [22]. Let $\mathscr{H}$ be a Hilbert space and let $T$ be selfadjoint operator on $\mathscr{H}$. For a subset $E \subset \operatorname{Sp} K$ we write $\mathscr{H}(T \in E)$ for the spectral subspace of $T$ corresponding to $E$. We extend this notation further by agreeing to write $\mathscr{H}(a \leq T \leq b)$ for $E=[a, b]$ and $\mathscr{H}(T=x)$ if $E=\{x\}$. Similarly we write $\mathscr{H}(T<r)$ for the spectral subspace corresponding to $]-\infty, r[$, etc.

Proof of Proposition 3.3. Let us note that due to (15) all operators involved preserve not only ker $K$, but also $(\operatorname{ker} K)^{\perp}$. Since on $\operatorname{ker} K$ formula (16) holds, we can immediately restrict to the subspace $\mathscr{H}=(\operatorname{ker} K)^{\perp}$. Note that this means that $\operatorname{ker} A^{*}=\operatorname{ker} K=\{0\}$.

Let $A=u|A|$ be the polar decomposition of $A$. Then $u$ is a partial isometry with initial subspace $(\operatorname{ker}|A|)^{\perp}=(\operatorname{ker}(K-\mathbb{1}))^{\perp}$ and final subspace $\overline{\operatorname{ran} A}=$ $\left(\operatorname{ker} A^{*}\right)^{\perp}=\mathscr{H}$. In other words $u$ is a co-isometry.

By the adjoint version of (15c) we have $K A^{*}=q^{2} A^{*} K$, so

$$
|A| K u^{*}=q^{2}|A| u^{*} K
$$

since $|A|$ commutes with $K$ by (15a). Moreover, as noted above, the range of $u^{*}$ is $(\operatorname{ker}|A|)^{\perp}$ and $K$ preserves this subspace. Therefore we may cancel $|A|$ in (17) to obtain

$$
q^{-2} K u^{*}=u^{*} K \text {. }
$$

Multiplying this relation from the right by $u$ we obtain that $q^{-2} K u^{*} u=u^{*} K u$ or

$$
q^{-2} K \psi=u^{*} K u \psi
$$

for any $\left.\psi \in(\operatorname{ker}|A|)^{\perp}=\operatorname{ker}(K-\mathbb{1})\right)^{\perp}$. Let us note the first consequence of (18). Namely let us take $\varepsilon>0$ and $\psi \in \mathscr{H}\left(q^{2}+\varepsilon \leq K \leq 1-\varepsilon\right)$. Then the left-hand side of (18) has norm greater or equal to $q^{-2}\left(q^{2}+\varepsilon\right)\|\psi\|=\left(1+q^{-2} \varepsilon\right)\|\psi\|$, while the norm of the right-hand side is smaller or equal to $\|\psi\|$. Therefore $\psi$ must be 0 . In other words, the spectral projection of $K$ corresponding to the interval $] q^{2}, 1[$ is zero. In other words,

$$
\operatorname{Sp} K \cap] q^{2}, 1[=\emptyset .
$$

Let us denote by $\mathcal{K}$ the space $(\operatorname{ker}(K-\mathbb{1}))^{\perp}=\operatorname{ran} u^{*}$. From (18) we gather that

$$
\left.q^{-2} K\right|_{\mathcal{K}}=\left.u^{*} K u\right|_{\mathcal{K}}
$$

Since $u$ is unitary from $\mathcal{K}$ onto $\mathscr{H}$ the spectrum of the right-hand side of (20) is equal to $\mathrm{Sp} K$. The spectrum of the left-hand side of (20) is $q^{-2}((\mathrm{Sp} K) \backslash\{1\})$. Taking into account (19) we find that

$$
\operatorname{Sp} K \subset\left\{1, q^{2}, q^{4}, \ldots\right\} \cup\{0\} .
$$


Let us decompose $\mathscr{H}$ into direct sum of eigenspaces of $K$ :

$$
\mathscr{H}=\bigoplus_{n=0}^{\infty} \mathscr{H}\left(K=q^{2 n}\right)
$$

In this decomposition we have

$$
\begin{aligned}
& K=\left[\begin{array}{lllll}
1 & & & \\
q^{2} & & & \\
& q^{4} & & \\
& & q^{6} & \\
& & & \ddots
\end{array}\right]
\end{aligned}
$$

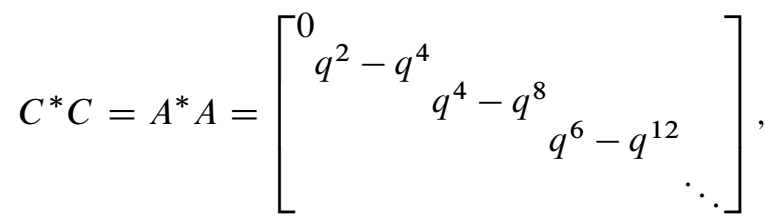

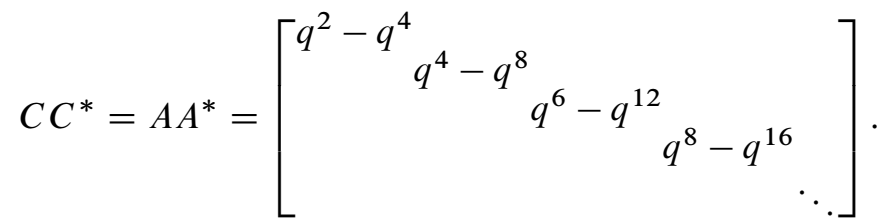

Let us also note that $u$ maps $\mathscr{H}(K=1)$ to $\{0\}$ and is an isometric map of $\mathscr{H}\left(K=q^{2 n}\right)$ onto $\mathscr{H}\left(K=q^{2 n-2}\right)$ for $n>0$. Also, since $|A|$ preserves the spectral subspaces of $K$ and is 0 on $\mathscr{H}(K=1)$ and invertible on the remaining ones, we see that $A$ maps $\mathscr{H}(K=1)$ to $\{0\}$ and

$$
A\left(\mathscr{H}\left(K=q^{2 n}\right)\right)=\mathscr{H}\left(K=q^{2 n-2}\right)
$$

for $n>0$.

Let us take $\psi \in \mathscr{H}\left(K=q^{2 n}\right)$ for some $n>0$. We have $C^{*} C \psi=\left(q^{2 n}-q^{4 n}\right) \psi$, so

$$
\left(q^{2 n}-q^{4 n}\right) C \psi=C C^{*} C \psi
$$

Thus $C \psi$ is an eigenvector of $C C^{*}$ with eigenvalue $q^{2 n}-q^{4 n}$. In other words

$$
C \psi \in \mathscr{H}\left(K=q^{2 n-2}\right) \oplus \mathscr{H}\left(K=1-q^{2 n-2}\right) .
$$

We will show that in fact

$$
C \psi \in \mathcal{H}\left(K=q^{2 n-2}\right) .
$$

Let us first notice that for generic $q$ the subspace $\mathscr{H}\left(K=1-q^{2 n-2}\right)$ is $\{0\}$ (e.g., for $q$ non algebraic or $q<\frac{\sqrt{2}}{2}$ ). Moreover we can assume that $1-q^{2 n-2} \neq q^{2 n-2}$ because in this particular case we already have (23) (and (22) must be modified accordingly). 
Assume that $n>1$. Taking into account (21) and (22) as well as (15d) we obtain

$$
\begin{aligned}
& A C \psi \in \mathscr{H}\left(K=q^{2 n-4}\right) \oplus \mathscr{H}\left(K=q^{-2}\left(1-q^{2 n-2}\right)\right) \\
& \| \\
& C A \psi \in \mathscr{H}\left(K=q^{2 n-4}\right) \oplus \mathscr{H}\left(K=1-q^{2 n-4}\right) .
\end{aligned}
$$

Since $1-q^{2 n-4} \neq q^{-2}-q^{2 n-4}$ we see that $A C \psi \in \mathscr{H}\left(K=q^{2 n-4}\right)$. Looking again at (21) and (22) gives (23).

Finally let us consider the case $n=1$. Here we have $\mathscr{H}\left(K=1-q^{2 n-2}\right)=$ $\mathscr{H}(K=0)=\{0\}$, so (23) follows from (22). Let $C=v|C|$ be the polar decomposition of $C$. Since $|C|$ preserves eigenspaces of $K$ we have

$$
\left.v \mathscr{H}\left(K=q^{2 n}\right)\right)=\mathscr{H}\left(K=q^{2 n-2}\right)
$$

for $n>0$.

Using (24) we compute for $\psi \in \mathscr{H}\left(K=q^{2 n}\right)$ with $n>0$ :

$$
\begin{aligned}
& K C \psi=K v|C| \psi=\left(q^{2 n}-q^{4 n}\right) K v \psi=\left(q^{2 n}-q^{4 n}\right) q^{2 n-2} v \psi, \\
& C K \psi=v|C| K \psi=q^{2 n} v|C| \psi=q^{2} n\left(q^{2 n}-q^{4 n}\right) v \psi,
\end{aligned}
$$

so that $C K=q^{2} K C$ on $\mathscr{H}(K<1)$. Clearly $C K \psi=q^{2} K C \psi=0$ for $\psi \in$ $\mathscr{H}(K=1)$. This finishes the proof of (16).

Proposition 3.3 shows that relation (14l) follows from (14e)-(14h), (14k) and (14p).

Remark 3.4. (1) For most values of $q$ Proposition 3.3 does not require assumption (15d). This is the case, e.g., for $q$ strictly smaller than $\frac{\sqrt{2}}{2}$ or for those $q$ for which $q^{2 n}+q^{2 m}$ is not equal to 1 for all $m, n \in \mathbb{N}$. In particular this is the case for non algebraic $q$.

However, if there exist $m_{0}, n_{0} \in \mathbb{N}$ such that $q^{2 n_{0}}=1-q^{2 m_{0}}$ and $n_{0} \neq m_{0}$ we can give an example of operators $A, C, K$ on a Hilbert space $H$ satisfying (15a)(15c) and failing (16). Indeed, let $H=\ell^{2}\left(\mathbb{Z}_{+}\right)$with standard orthonormal basis $\left(e_{n}\right)_{n \in \mathbb{Z}_{+}}$. Let

$$
K e_{n}=q^{2 n} e_{n}, \quad s e_{n}=\left\{\begin{array}{ll}
0 & n=0, \\
e_{n-1} & n>0,
\end{array} \quad \sigma e_{n}= \begin{cases}e_{n} & n \neq n_{0}, m_{0}, \\
e_{m_{0}} & n=n_{0}, \\
e_{n_{0}} & n=m_{0} .\end{cases}\right.
$$

Now putting $K=s \sqrt{K=K^{2}}$ and $C=s \sigma \sqrt{K-K^{2}}$ we obtain the required example.

(2) Let us note that Proposition 3.3 is not true for $q=1$. Indeed one can take $H=L^{2}([0,1])$ and $K$ the multiplication by the identity function on $[0,1]$. Let $A=\sqrt{K-K^{2}}$. Moreover let $u$ be the unitary map on $H$ induced by the flip

$$
[0,1] \ni t \longmapsto 1-t \in[0,1]
$$


and let $C=u A$. Then $A K=K A$, but $C K=(\mathbb{1}-K) C \neq K C$. Moreover we have $A C=C A$.

3.2. Action on $\boldsymbol{Q S}\left(\boldsymbol{M}_{2}\right)$. Let us fix $\left.q \in\right] 0,1\left[\right.$. The quantum group $\mathrm{S}_{q} \mathrm{O}(3)$ acts on the quantum space $\mathcal{Q S}\left(M_{2}\right)$. The action is described by the morphism $\Psi_{q} \in$ $\operatorname{Mor}\left(M_{2}, M_{2} \otimes \mathrm{C}\left(\mathrm{S}_{q} \mathrm{O}(3)\right)\right)$,

$$
\Psi_{q}(\boldsymbol{n})=\left[\begin{array}{cc}
-q A & L \\
-q G & q^{-1} A
\end{array}\right] .
$$

This action comes from the action of $\mathrm{S}_{q} \mathrm{U}(2)$ on $\mathcal{Q S}\left(M_{2}\right)$ induced by the fundamental representation of $\mathrm{S}_{q} \mathrm{U}(2)$ and is therefore continuous ([6], Lemma 2.1).

One can check using relations (14) that the action of $\mathrm{S}_{q} \mathrm{O}(3)$ preserves the state $\omega_{q}$ introduced by (6). Therefore there exists a unique map $\Lambda_{q} \in \operatorname{Mor}\left(\boldsymbol{A}, \mathrm{C}\left(\mathrm{S}_{q} \mathrm{O}(3)\right)\right)$ such that

$$
\Psi_{q}=\left(\operatorname{id}_{M_{2}} \otimes \Lambda_{q}\right) \circ \Phi
$$

In particular we have

$$
\Lambda_{q}(\beta)=L, \quad \Lambda_{q}(\gamma)=-q G, \quad \Lambda_{q}(\delta)=q^{-1} A .
$$

The symbols

$$
\mathrm{C}\left(\mathrm{S}_{q} \mathrm{O}(3)\right), \quad \Delta_{q}, \quad \Psi_{q}, \quad \Lambda_{q}
$$

will be used throughout the paper in the meaning introduced above.

\section{Characterization of quantum SO(3) groups}

Let $\mathscr{E}=\left(\mathfrak{B}, \Delta_{\mathfrak{B}}\right)$ be a compact quantum group with a given continuous action $\Psi_{\mathfrak{B}}$ on $\mathcal{Q S}\left(M_{2}\right)$, i.e., $\Psi_{\mathfrak{B}} \in \operatorname{Mor}\left(M_{2}, M_{2} \otimes \mathfrak{B}\right)$ and

$$
\left(\Psi_{\mathfrak{B}} \otimes \operatorname{id}_{\mathfrak{B}}\right) \circ \Psi_{\mathfrak{B}}=\left(\operatorname{id}_{M_{2}} \otimes \Delta_{\mathfrak{B}}\right) \circ \Psi_{\mathfrak{B}} .
$$

Assume further that the action of $\mathcal{E}$ preserves the state $\omega_{q}$. Then using the notation introduced in Section 2.2 there is a unique $\Lambda \in \operatorname{Mor}(\boldsymbol{A}, \mathfrak{B})$ satisfying

$$
\Psi_{\mathfrak{B}}=\left(\operatorname{id}_{M_{2}} \otimes \Lambda\right) \circ \boldsymbol{\Phi} .
$$

Note that this implies that

$$
\Psi_{\mathfrak{B}}(\boldsymbol{n})=\left(\mathrm{id}_{M_{2}} \otimes \Lambda\right)\left[\begin{array}{cc}
-q^{2} \delta & \beta \\
\gamma & \delta
\end{array}\right] .
$$

We will prove that $\Lambda$ factorizes uniquely through $\Lambda_{q} \in \operatorname{Mor}\left(\boldsymbol{A}, \mathrm{C}\left(\mathrm{S}_{q} \mathrm{O}(3)\right)\right)$ introduced in Section 3.2. Let us denote by $\mathscr{B}$ the canonical Hopf *-algebra dense in $\mathscr{B}$ and let $\epsilon_{\mathscr{B}}$ and $\kappa_{\mathscr{B}}$ be its counit and antipode ([21], Section 2). 
Proposition 4.1. Let

$$
b=\Lambda(\beta), \quad c=\Lambda(\gamma), \quad d=\Lambda(\delta) .
$$

Then

$$
\begin{aligned}
& q^{4} d^{*} d+c^{*} c+q^{4} d d^{*}+b b^{*}=\mathbb{1}, \\
& b^{*} b+d^{*} d+c c^{*}+d d^{*}=\mathbb{1},
\end{aligned}
$$

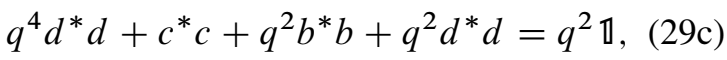

$$
\begin{aligned}
& q^{4} d d^{*}+b b^{*}+q^{2} c c^{*}+q^{2} d d^{*}=\mathbb{1}, \\
& c^{*} d-q^{2} d^{*} b+b d^{*}-q^{2} d c^{*}=0, \\
& b c=-q^{4} d^{2}, \\
& c b=-d^{2} \text {, } \\
& b d=q^{2} d b, \\
& d c=q^{2} c d \text {. }
\end{aligned}
$$

Moreover $b, c, d \in \mathscr{B}$ and we have

$$
\epsilon_{\mathcal{B}}(c)=\epsilon_{\mathscr{B}}(d)=0 \text { and } \epsilon_{\mathscr{B}}(b)=1 .
$$

Proof. Relations (29) are consequences of relations (7) and (8) satisfied by $\beta, \gamma$ and $\delta$. By results of [7], Theorem 3.6, [10], Theorem 1.5, and [11], Theorem 6.3, we know that there is a dense "smooth" subalgebra $\mathcal{M}$ of $M_{2}$ such that $\Psi_{\mathcal{B}}$ restricted to this subalgebra is a right coaction of the Hopf ${ }^{*}$-algebra $\mathcal{B}$ on $\mathcal{M}$. Clearly $\mathcal{M}=M_{2}$ and thus $b, c, d \in \mathscr{B}$ and $\epsilon_{\mathscr{B}}(c)=\epsilon_{\mathscr{B}}(d)=0, \epsilon_{\mathscr{B}}(b)=1$.

We will keep the notation $b, c$ and $d$ for images of $\beta, \gamma$ and $\delta$ under $\Lambda$ throughout the paper. In terms of these elements we have (cf. (28))

$$
\Psi_{\mathfrak{B}}(\boldsymbol{n})=\left[\begin{array}{cc}
-q^{2} d & b \\
c & d
\end{array}\right]
$$

Proposition 4.2. Let

$$
\boldsymbol{a}=\left[\begin{array}{cccc}
q^{4} d d^{*}+b b^{*} & -q d & -q^{2} d^{*} & q^{3} d^{*} d+q^{-1} c^{*} c \\
q b d^{*}-q^{3} d c^{*} & b & q c^{*} & c^{*} d-q^{2} d^{*} b \\
d b^{*}-q^{2} c d^{*} & q^{-1} c & b^{*} & q^{-1} d^{*} c-q b^{*} d \\
q c c^{*}+q d d^{*} & d & q d^{*} & b^{*} b+d^{*} d
\end{array}\right] .
$$

Then the matrix $\boldsymbol{a} \in M_{4}(\mathfrak{B})$ is unitary.

Proof. Let $a_{i, j}$ be the $(i, j)$-entry of $\boldsymbol{a}$ and define

$$
\begin{array}{ll}
e_{1}=\sqrt{1+q^{2}} \boldsymbol{n} \boldsymbol{n}^{*}, & e_{2}=\frac{\sqrt{1+q^{2}}}{q} \boldsymbol{n}, \\
e_{3}=\sqrt{1+q^{2}} \boldsymbol{n}^{*}, & e_{4}=\frac{\sqrt{1+q^{2}}}{q} \boldsymbol{n}^{*} \boldsymbol{n} .
\end{array}
$$


One can check that

$$
\Psi_{\mathfrak{B}}\left(e_{j}\right)=\sum_{i=1}^{4} e_{i} \otimes a_{i, j}
$$

and from this and (27) it follows that

$$
\Delta_{\mathfrak{B}}\left(a_{i, j}\right)=\sum_{k=1}^{4} a_{i, k} \otimes a_{k, j}
$$

Moreover, since $\left\{e_{1}, \ldots, e_{4}\right\}$ is an orthonormal basis of $M_{2}$ for the scalar product $\left(m_{1} \mid m_{2}\right)=\omega_{q}\left(m_{1}^{*} m_{2}\right)$ for $m_{1}, m_{2} \in M_{2}$, we have

$$
a^{*} a=\mathbb{1}
$$

(cf. [14], Proof of Theorem 7.3). To prove that $\boldsymbol{a}$ is unitary it is therefore enough to show that $\boldsymbol{a}$ is right invertible. We know that the entries of $\boldsymbol{a}$ belong to $\mathcal{B}$. In particular we can apply $\kappa_{\mathscr{B}}$ to these elements. If $\boldsymbol{b}$ is a matrix with entries $\kappa_{\mathcal{B}}\left(a_{i, j}\right)$ then $\boldsymbol{a} \boldsymbol{b}$ is (by (32)) a matrix with $\epsilon_{\mathcal{B}}\left(a_{i, j}\right) \mathbb{1}$ as the $(i, j)$-entry. It follows from (30) that $\boldsymbol{a}$ is invertible.

Theorem 4.3. There exists a unique $\Gamma \in \operatorname{Mor}\left(\mathrm{C}\left(\mathrm{S}_{q} \mathrm{O}(3)\right)\right.$, $\left.\mathfrak{B}\right)$ such that $\Lambda=\Gamma \circ \Lambda_{q}$.

Proof. Let

$$
\dot{A}=q d, \dot{C}=b d^{*}-q^{2} d c^{*}, \dot{G}=-q^{-1} c, \dot{K}=q^{2} d^{*} d+q^{-2} c^{*} c, \dot{L}=b .
$$

Using relations (29a), (29c), (29d) and (29e) one can show that the matrix $\boldsymbol{a}$ defined in Proposition 4.2 can be expressed using $\dot{A}, \dot{C}, \dot{G}, \dot{K}$ and $\dot{L}$ :

$$
\boldsymbol{a}=\left[\begin{array}{cccc}
\mathbb{1}-q^{2} \dot{K} & -\dot{A} & -q \dot{A}^{*} & q \dot{K} \\
q \dot{C} & \dot{L} & -q^{2} \dot{G}^{*} & -\dot{C} \\
\dot{C}^{*} & -\dot{G} & \dot{L}^{*} & -q^{-1} \dot{C}^{*} \\
q \dot{K} & q^{-1} \dot{A} & \dot{A}^{*} & \mathbb{1}-\dot{K}
\end{array}\right]
$$

Our aim is to use the unitarity of $\boldsymbol{a}$ and the remaining relations (29) to show that the elements (33) satisfy the Podleś relations (14).

By the results of Section 3.1 we do not need to check relations (14j), (14q) and (14t). Moreover (14m), (14n), (14o) and (14r) follow immediately from (29f)-(29g). Relation (14l) will be shown the moment we verify (14e)-(14h), (14k) and (14p), by Proposition 3.3.

To make calculations easier let us list some of the relations following from the unitarity of $\boldsymbol{a}$. Considering the $(1,2),(1,3),(2,2),(3,3)(4,3)$ and $(4,4)$ entries of 
$\boldsymbol{a} \boldsymbol{a}^{*}$ we obtain

$$
\begin{aligned}
q\left(\mathbb{1}-q^{2} \dot{K}\right) \dot{C}^{*}-\dot{A} \dot{L}^{*}+q^{3} \dot{A}^{*} \dot{G}-q \dot{K} \dot{C}^{*} & =0, \\
\left(\mathbb{1}-q^{2} \dot{K}\right) \dot{C}+\dot{A} \dot{G}^{*}-q \dot{A}^{*} \dot{L}-\dot{K} \dot{C} & =0, \\
q^{2} \dot{C} \dot{C}^{*}+\dot{L} \dot{L}^{*}+q^{4} \dot{G}^{*} \dot{G}+\dot{C} \dot{C}^{*} & =\mathbb{1}, \\
\dot{C}^{*} \dot{C}+\dot{G} \dot{G}^{*}+\dot{L}^{*} \dot{L}+q^{-2} \dot{C} \dot{C}^{*} & =\mathbb{1}, \\
q \dot{K} \dot{C}-q^{-1} \dot{A} \dot{G}^{*}+\dot{A}^{*} \dot{L}-q^{-1}(\mathbb{1}-\dot{K}) \dot{C} & =0, \\
q^{2} \dot{K}^{2}+q^{-2} \dot{A} \dot{A}^{*}+\dot{A}^{*} \dot{A}+(\mathbb{1}-\dot{K})^{2} & =\mathbb{1} .
\end{aligned}
$$

Similarly considering the $(2,2)$ and $(4,4)$ entries of $\boldsymbol{a}^{*} \boldsymbol{a}$ we obtain

$$
\begin{aligned}
\dot{A}^{*} \dot{A}+\dot{L}^{*} \dot{L}+\dot{G}^{*} \dot{G}+q^{-2} \dot{A}^{*} \dot{A} & =\mathbb{1} \\
q^{2} \dot{K}^{2}+\dot{C}^{*} \dot{C}+q^{-2} \dot{C} \dot{C}^{*}+(\mathbb{1}-\dot{K})^{2} & =\mathbb{1} .
\end{aligned}
$$

Finally let us rewrite (29b) in terms of elements (33):

$$
\dot{L}^{*} \dot{L}+q^{-2} \dot{A}^{*} \dot{A}+q^{2} \dot{G} \dot{G}^{*}+q^{-2} \dot{A} \dot{A}^{*}=\mathbb{1} .
$$

Step 1: normality of $\dot{G}$. The matrix $\boldsymbol{a} \in M_{4}(\mathscr{B})$ is unitary and its inverse is $\left(\operatorname{id}_{M_{4}} \otimes \kappa_{B}\right) a$. Therefore we know the values of $\kappa_{\mathcal{B}}$ on matrix elements of (34):

$$
\begin{aligned}
& \kappa_{\mathscr{B}}(\dot{A})=-q \dot{C}^{*}, \quad \kappa_{B}\left(\dot{A}^{*}\right)=-q^{-1} \dot{C}, \\
& \kappa_{\mathscr{B}}(\dot{C})=-q^{-1} \dot{A}^{*}, \quad \kappa_{\mathscr{B}}\left(\dot{C}^{*}\right)=-q \dot{A}, \\
& \kappa_{\mathscr{B}}(\dot{G})=q^{2} \dot{G}, \quad \kappa_{\mathscr{B}}\left(\dot{G}^{*}\right)=q^{-2} \dot{G}^{*} \text {, } \\
& \kappa_{\mathscr{B}}(\dot{L})=\dot{L}^{*}, \quad \kappa_{\mathscr{B}}\left(\dot{L}^{*}\right)=\dot{L}, \\
& \kappa_{\mathscr{B}}(\dot{K})=\dot{K} \text {. }
\end{aligned}
$$

By antimultiplicativity of $\kappa_{\mathscr{B}}$ we have

$$
\kappa_{\mathcal{B}}\left(\dot{G}^{*} \dot{G}\right)=\dot{G} \dot{G}^{*} \text { and } \kappa_{\mathcal{B}}\left(\dot{A}^{*} \dot{A}\right)=\dot{C}^{*} \dot{C}
$$

Therefore

$$
\dot{A}^{*} \dot{A}+\dot{G}^{*} \dot{G}=\dot{K}=\kappa_{\mathcal{B}}(\dot{K})=\dot{C}^{*} \dot{C}+\dot{G} \dot{G}^{*} .
$$

Thus, by (36a) and (35d) we have

$$
\dot{A}^{*} \dot{A}=\dot{C}^{*} \dot{C},
$$

and so, by (38) again we have

$$
\dot{G}^{*} \dot{G}=\dot{G} \dot{G}^{*}
$$

We have thus checked the relation (14c). 
Step 2: consequences. Now we note that normality of $\dot{G}$ implies additional commutation relations with $\dot{L}$ and $\dot{A}$. By Theorem 1.2 we have

$$
\begin{aligned}
& \dot{L} \dot{G}^{*}=q^{4} \dot{G}^{*} \dot{L}, \\
& \dot{A} \dot{G}^{*}=q^{2} \dot{G}^{*} \dot{A} .
\end{aligned}
$$

Moreover (36b) and (35f) together with (39) give

$$
\dot{C} \dot{C}^{*}=\dot{A} \dot{A}^{*} .
$$

From (36b) we have

$$
\dot{A}^{*} \dot{A}+q^{-2} \dot{A} \dot{A}^{*}=2 \dot{K}-\dot{K}^{2}-q^{2} \dot{K}^{2},
$$

while inserting (39) into (35d) and using (37) gives

$$
\dot{A}^{*} \dot{A}-q^{-2} \dot{A} \dot{A}^{*}=\left(q^{2}-1\right) \dot{G} \dot{G}^{*}=\left(q^{2}-1\right) \dot{G}^{*} \dot{G} .
$$

Adding (44) and (43) leads to

$$
2 \dot{A}^{*} \dot{A}=q^{2} \dot{G}^{*} \dot{G}-q^{2} \dot{K}^{2}+2 \dot{K}-\dot{G}^{*} \dot{G}-\dot{K}^{2} .
$$

Since $\dot{K}=\dot{G}^{*} \dot{G}+\dot{A}^{*} \dot{A}$, from (45) we get

$$
\dot{K}^{2}=\dot{G}^{*} \dot{G},
$$

so (14d) is satisfied. Consequently (45) gives

$$
\dot{A}^{*} \dot{A}=\dot{K}-\dot{K}^{2}
$$

and thus (14e) and (14g) are checked. Now inserting (47) into (44) quickly yields

$$
\dot{A} \dot{A}^{*}=q^{2} \dot{K}-q^{4} \dot{K}^{2},
$$

so we get (14f) and (by (42)) (14h).

Inserting (46), (47) and (48) into (37) gives

$$
\dot{L}^{*} \dot{L}=(\mathbb{1}-\dot{K})\left(\mathbb{1}-q^{-2} \dot{K}\right) .
$$

Similarly, inserting (42) into (35c) and using (47) and (48), we obtain that

$$
\dot{L} \dot{L}^{*}=\left(\mathbb{1}-q^{2} \dot{K}\right)\left(I-q^{4} \dot{K}\right) .
$$

This means that we checked relations (14a) and (14b).

Step 3: commutation of $\dot{A}$ and $\dot{C}$. By (33) we have

$$
\dot{C}=q^{-1} \dot{L} \dot{A}^{*}+q^{2} \dot{A} \dot{G}^{*} .
$$


Therefore, using (44), (41b), (40) and analogs for $\dot{A}, \dot{G}$ and $\dot{L}$ of relations (14n) and (14r) we compute

$$
\begin{aligned}
\dot{C} \dot{A} & =\left(q^{-1} \dot{L} \dot{A}^{*}+q^{2} \dot{A} \dot{G}^{*}\right) \dot{A} \\
& =q^{-1} \dot{L} \dot{A}^{*} \dot{A}+q^{2} \dot{A} \dot{G}^{*} \dot{A} \\
& =q^{-1} \dot{L}\left(q^{-2} \dot{A} \dot{A}^{*}+\left(q^{2}-1\right) \dot{G}^{*} \dot{G}\right)+q^{2} \dot{A} \dot{G}^{*} \dot{A} \\
& =q^{-3} \dot{L} \dot{A} \dot{A}^{*}+\left(q-q^{-1}\right) \dot{L} \dot{G}^{*} \dot{G}+\dot{A}^{2} \dot{G}^{*} \\
& =q^{-3} \dot{L} \dot{A} \dot{A}^{*}+\left(q-q^{-1}\right) \dot{L} \dot{G} \dot{G}^{*}+\dot{A}^{2} \dot{G}^{*} \\
& =q^{-1} \dot{A} \dot{L} \dot{A}^{*}+\left(q-q^{-1}\right) \dot{L} \dot{G} \dot{G}^{*}+\dot{A}^{2} \dot{G}^{*} \\
& =q^{-1} \dot{A} \dot{L} \dot{A}^{*}+\left(q^{2}-1\right) \dot{A}^{2} \dot{G}^{*}+\dot{A}^{2} \dot{G}^{*} \\
& =q^{-1} \dot{A} \dot{L} \dot{A}^{*}+q^{2} \dot{A}^{2} \dot{G}^{*}=\dot{A} \dot{C},
\end{aligned}
$$

which satisfies relation (14p).

Step 4: finishing touches. Let us now address relation (14k). By (44) and (41b) we have

$$
\begin{aligned}
\dot{K} \dot{A} & =\left(\dot{A}^{*} \dot{A}+\dot{G}^{*} \dot{G}\right) \dot{A} \\
& =\dot{A}^{*} \dot{A} \dot{A}+\dot{G}^{*} \dot{G} \dot{A} \\
& =\left(q^{-2} \dot{A} \dot{A}^{*}+\left(q^{2}-1\right) \dot{G}^{*} \dot{G}\right) \dot{A}+\dot{G}^{*} \dot{G} \dot{A} \\
& =q^{-2} \dot{A} \dot{A}^{*} \dot{A}+\left(q^{2}-1\right) \dot{G}^{*} \dot{G} \dot{A}+\dot{G}^{*} \dot{G} \dot{A} \\
& =q^{-2} \dot{A} \dot{A}^{*} \dot{A}+q^{2} \dot{G}^{*} \dot{G} \dot{A} \\
& =q^{-2} \dot{A} \dot{A}^{*} \dot{A}+q^{-2} \dot{A} \dot{G}^{*} \dot{G}=q^{-2} \dot{A} \dot{K} .
\end{aligned}
$$

In addition to (14k) we now also have (14l) (cf. Proposition 3.3).

Let us rewrite (35a) and (35b) using (41b) as

$$
\begin{array}{r}
\dot{C}-q^{2} \dot{C} \dot{K}-q^{-1} \dot{L} \dot{A}^{*}+q^{2} \dot{G}^{*} \dot{A}-\dot{C} \dot{K}=0, \\
\dot{C}-q^{2} \dot{K} \dot{C}-\dot{A}^{*} \dot{L}+\dot{A} \dot{G}^{*}-\dot{K} \dot{C}=0 .
\end{array}
$$

Consequently

$$
q^{2}(\dot{K} \dot{C}-\dot{C} \dot{K})+q \dot{A}^{*} \dot{L}-q^{-1} \dot{L} \dot{A}^{*}+(\dot{K} \dot{C}-\dot{C} \dot{K})=0 .
$$

Now note that

$$
q^{-1} \dot{L} \dot{A}^{*}+q^{2} \dot{A} \dot{G}^{*}=\dot{C}=q \dot{A}^{*} \dot{L}+\dot{G}^{*} \dot{A}
$$

by (33) and (29e). Therefore, using (41b), we obtain

$$
q^{-1} \dot{L} \dot{A}^{*}-q \dot{A}^{*} \dot{L}=\left(1-q^{4}\right) \dot{G}^{*} \dot{A} .
$$

Thus (49) can be rewritten as

$$
\left(1+q^{2}\right)(\dot{K} \dot{C}-\dot{C} \dot{K})=\left(1-q^{4}\right) \dot{G}^{*} \dot{A} .
$$


Now using (14l) we obtain $\dot{K} \dot{C}=\dot{G}^{*} \dot{A}$ and again by (41b) we get

$$
q^{-1} \dot{A} \dot{G}^{*}=q \dot{K} \dot{C}
$$

Plugging this into (35e) gives

$$
\dot{A}^{*} \dot{L}=q^{-1}(\mathbb{1}-\dot{K}) \dot{C},
$$

so (14s) is satisfied.

The last relation from the list (14) that remains to be checked is (14i). For this purpose, using (49), (41a), (41b), (14r), (14n) and (14m), we compute

$$
\begin{aligned}
\dot{L} \dot{K} & =\dot{L}\left(\dot{A}^{*} \dot{A}+\dot{G}^{*} \dot{G}\right)=\dot{L} \dot{A}^{*} \dot{A}+\dot{L} \dot{G}^{*} \dot{G} \\
& =\left(q^{2} \dot{A}^{*} \dot{L}+q \dot{G}^{*} \dot{A}-q^{3} \dot{A} \dot{G}^{*}\right) \dot{A}+\dot{L} \dot{G}^{*} \dot{G} \\
& =q^{2} \dot{A}^{*} \dot{L} \dot{A}+q \dot{G}^{*} \dot{A} \dot{A}-q^{3} \dot{A} \dot{G}^{*} \dot{A}+\dot{L} \dot{G}^{*} \dot{G} \\
& =q^{2} \dot{A}^{*} \dot{L} \dot{A}+q \dot{G}^{*} \dot{A} \dot{A}-q^{3} \dot{A} \dot{G}^{*} \dot{A}+q^{4} \dot{G}^{*} \dot{L} \dot{G} \\
& =q^{2} \dot{A}^{*} \dot{L} \dot{A}+q \dot{G}^{*} \dot{A} \dot{A}-q^{5} \dot{G}^{*} \dot{A} \dot{A}+q^{4} \dot{G}^{*} \dot{L} \dot{G} \\
& =q^{2} \dot{A}^{*} \dot{L} \dot{A}+\dot{G}^{*} \dot{L} \dot{G}-q^{4} \dot{G}^{*} \dot{L} \dot{G}+q^{4} \dot{G}^{*} \dot{L} \dot{G} \\
& =q^{4} \dot{A}^{*} \dot{A} \dot{L}+q^{4} \dot{G}^{*} \dot{G} \dot{L}=q^{4} \dot{K} \dot{L},
\end{aligned}
$$

which means that $\dot{A}, \dot{C}, \dot{G}, \dot{K}$ and $\dot{L}$ satisfy all relations from the list (14). Therefore there exists a unique $\Gamma: \mathrm{C}\left(\mathrm{S}_{q} \mathrm{O}(3)\right) \rightarrow \mathfrak{B}$ such that

$$
\Gamma(A)=\dot{A}, \quad \Gamma(C)=\dot{C}, \quad \Gamma(G)=\dot{G}, \quad \Gamma(K)=\dot{K}, \quad \Gamma(L)=\dot{L} .
$$

By (26) we have $\Gamma \circ \Lambda_{q}=\Lambda$. Since $\mathrm{C}\left(\mathrm{S}_{q} \mathrm{O}(3)\right)$ is generated by $A, G$ and $L$, this condition determines $\Gamma$ uniquely.

Corollary 4.4. Let $\Gamma \in \operatorname{Mor}\left(\mathrm{C}\left(\mathrm{S}_{q} \mathrm{O}(3)\right)\right.$, $\left.\mathfrak{B}\right)$ be the map defined in Theorem 4.3. Then

(1) $\left(\operatorname{id}_{M_{2}} \otimes \Gamma\right) \circ \Psi_{q}=\Psi_{\mathfrak{B}}$ and this property determines $\Gamma$ uniquely,

(2) $\Delta_{\mathscr{B}} \circ \Gamma=(\Gamma \otimes \Gamma) \circ \Delta_{q}$.

Proof. The formula from statement (1) follows easily from (25) and (31). Note that applying this formula to the generator $\boldsymbol{n} \in M_{2}$ and comparing matrix elements fixes $\Gamma$ on elements $A, G, L$ generating $\mathrm{C}\left(\mathrm{S}_{q} \mathrm{O}(3)\right.$ ) (cf. Remark $3.2(1)$ ). 
To prove the second statement we compute

$$
\begin{aligned}
\left.\left(\operatorname{id}_{M_{2}} \otimes\left[(\Gamma \otimes \Gamma) \circ \Delta_{q}\right)\right]\right) \circ \Psi_{q} & =\left(\operatorname{id}_{M_{2}} \otimes \Gamma \otimes \Gamma\right) \circ\left(\operatorname{id}_{M_{2}} \otimes \Delta_{q}\right) \circ \Psi_{q} \\
& =\left(\operatorname{id}_{M_{2}} \otimes \Gamma \otimes \Gamma\right) \circ\left(\Psi_{q} \otimes \operatorname{id}_{\mathrm{C}\left(\mathrm{S}_{q} \mathrm{O}(3)\right)}\right) \circ \Psi_{q} \\
& =\left(\left[\left(\operatorname{id}_{M_{2}} \otimes \Gamma\right) \circ \Psi_{q}\right] \otimes \Gamma\right) \circ \Psi_{q} \\
& =\left(\Psi_{\mathfrak{B}} \otimes \mathrm{id}_{\mathfrak{B}}\right) \circ \Psi_{\mathfrak{B}} \\
& =\left(\operatorname{id}_{M_{2}} \otimes \Delta_{\mathfrak{B}}\right) \circ \Psi_{\mathfrak{B}} \\
& =\left(\operatorname{id}_{M_{2}} \otimes \Delta_{\mathfrak{B}}\right) \circ\left(\mathrm{id}_{M_{2}} \otimes \Gamma\right) \circ \Psi_{q} \\
& =\left(\operatorname{id}_{M_{2}} \otimes\left[\Delta_{\mathfrak{B}} \circ \Gamma\right]\right) \circ \Psi_{q} .
\end{aligned}
$$

Again applying both sides of (50) to $n$ and comparing matrix elements yields statement (2).

We have thus proved Theorem 1.1 for $q \in] 0,1[$.

Remark 4.5. Let $u$ be the fundamental representation of the quantum group $\mathrm{S}_{q} \mathrm{U}(2)$ ([18], Theorem 1.4 and §5). Then the tensor product $S=u$ (T) $u$ (cf. [19], p. 632) is a four-dimensional representation of $\mathrm{S}_{q} \mathrm{U}(2)$ which factorizes through $\mathrm{S}_{q} \mathrm{O}(3)$. In other words its matrix elements belong to the $\mathrm{C}^{*}$-subalgebra $\left(\mathrm{S}_{q} \mathrm{O}(3)\right)$ of $\mathrm{C}\left(\mathrm{S}_{q} \mathrm{U}(2)\right)$. Conjugating this matrix with

$$
V=\left[\begin{array}{cccc}
0 & -1 & 0 & 0 \\
1 & 0 & 0 & 0 \\
0 & 0 & 0 & -1 \\
0 & 0 & 1 & 0
\end{array}\right]
$$

we obtain

$$
V S V^{*}=\left[\begin{array}{cccc}
1-q^{2} K & -A & -q A^{*} & q K \\
q C & L & -q^{2} G^{*} & -C \\
C^{*} & -G & L^{*} & -q^{-1} C^{*} \\
q K & q^{-1} A & A^{*} & 1-K
\end{array}\right]
$$

(cf. (34)).

\section{Cases of $q=1$ and $q=0$}

5.1. Case $q=1$. The state $\omega_{q}$ on $M_{2}$ becomes the normalized trace tr when we put $q=1$. In this subsection we will show that the universal compact quantum group acting on $M_{2}$ and preserving the trace is the classical group $\mathrm{SO}(3)$. This is contrary to [16], Remark on p. 203. Although for $q=1$ the quantum group $\mathrm{S}_{q} \mathrm{O}(3)$ is isomorphic to the classical $\mathrm{SO}(3)$ ([8], Remark 3), we cannot follow directly the path of Section 4 because some of the tools we used there are no longer applicable to the case $q=1$ (e.g., Proposition 3.3, cf. Remark 3.4(2)).

The description of $Q-\operatorname{Map}^{\text {tr }}\left(\mathcal{Q S}\left(M_{2}\right)\right)$ is the following. 
Proposition 5.1. Let $\mathrm{Q}_{-\mathrm{Map}^{\mathrm{tr}}}\left(\mathcal{Q S}\left(M_{2}\right)\right)=(\boldsymbol{A}, \boldsymbol{\Delta})$. Then $\boldsymbol{A}$ is the universal $C^{*_{-}}$ algebra generated by three elements $\beta, \gamma$ and $\delta$ satisfying

$$
\begin{array}{rlrl}
\delta^{*} \delta+\gamma^{*} \gamma+\delta \delta^{*}+\beta \beta^{*} & =\mathbb{1}, & & \beta \gamma=-\delta^{2}, \\
\beta^{*} \beta+\delta^{*} \delta+\gamma \gamma^{*}+\delta \delta^{*} & =\mathbb{1}, & & \gamma \beta=-\delta^{2}, \\
\delta^{*} \delta+\gamma^{*} \gamma+\beta^{*} \beta+\delta^{*} \delta & =\mathbb{1}, & & \beta \delta=\delta \beta, \\
\delta \delta^{*}+\beta \beta^{*}+\gamma \gamma^{*}+\delta \delta^{*} & =\mathbb{1}, & & \delta \gamma=\gamma \delta, \\
\gamma^{*} \delta-\delta^{*} \beta+\beta \delta^{*}-\delta \gamma^{*} & =0 . &
\end{array}
$$

The comultiplication $\boldsymbol{\Delta} \in \operatorname{Mor}(\boldsymbol{A}, \boldsymbol{A} \otimes \boldsymbol{A})$ is

$$
\begin{aligned}
& \boldsymbol{\Delta}(\beta)=\delta \gamma^{*} \otimes \delta-\beta \delta^{*} \otimes \delta+\beta \otimes \beta+\gamma^{*} \otimes \gamma-\delta^{*} \beta \otimes \delta+\gamma^{*} \delta \otimes \delta, \\
& \boldsymbol{\Delta}(\gamma)=\gamma \delta^{*} \otimes \delta-\delta \beta^{*} \otimes \delta+\gamma \otimes \beta+\beta^{*} \otimes \gamma-\beta^{*} \delta \otimes \delta+\delta^{*} \gamma \otimes \delta, \\
& \boldsymbol{\Delta}(\delta)=-\gamma^{*} \gamma \otimes \delta-\delta \delta^{*} \otimes \delta+\delta \otimes \beta+\delta^{*} \otimes \gamma+\beta^{*} \beta \otimes \delta+\delta^{*} \delta \otimes \delta .
\end{aligned}
$$

The action of $\mathcal{Q}-\operatorname{Map}^{\mathrm{tr}}\left(\mathcal{Q S}\left(M_{2}\right)\right)$ on $\mathcal{Q S}\left(M_{2}\right)$ is given by $\boldsymbol{\Phi} \in \operatorname{Mor}\left(M_{2}, M_{2} \otimes \boldsymbol{A}\right)$ defined by

$$
\boldsymbol{\Phi}(\boldsymbol{n})=\left[\begin{array}{cc}
-\delta & \beta \\
\gamma & \delta
\end{array}\right] .
$$

The counit $\epsilon$ maps $\gamma$ and $\delta$ to 0 and $\beta$ to 1 .

Let $\mathcal{E}=\left(\mathfrak{B}, \Delta_{\mathfrak{B}}\right)$ be a compact quantum group acting on $M_{2}$ preserving the state tr and let $\Psi_{\mathscr{B}} \in \operatorname{Mor}\left(M_{2}, M_{2} \otimes \mathfrak{B}\right)$ be this action. There exists a unique $\Lambda \in \operatorname{Mor}(\boldsymbol{A}, \mathfrak{B})$ such that $\left(\operatorname{id}_{M_{2}} \otimes \Lambda\right) \circ \boldsymbol{\Phi}=\Psi_{\mathfrak{B}}$. As in Proposition 4.1 we let

$$
b=\Lambda(\beta), \quad c=\Lambda(\gamma), \quad d=\Lambda(\delta) .
$$

Then we have

$$
\Psi_{\mathfrak{B}}(\boldsymbol{n})=\left[\begin{array}{cc}
-d & b \\
c & d
\end{array}\right]
$$

and

$$
\begin{aligned}
d^{*} d+c^{*} c+d d^{*}+b b^{*} & =\mathbb{1}, & b c & =-d^{2}, \\
b^{*} b+d^{*} d+c c^{*}+d d^{*} & =\mathbb{1}, & c b & =-d^{2}, \\
d^{*} d+c^{*} c+b^{*} b+d^{*} d & =\mathbb{1}, & b d & =d b, \\
d d^{*}+b b^{*}+c c^{*}+d d^{*} & =\mathbb{1}, & d c & =c d, \\
c^{*} d-d^{*} b+b d^{*}-d c^{*} & =0 . & &
\end{aligned}
$$

Moreover, the matrix

$$
\left[\begin{array}{cccc}
d d^{*}+b b^{*} & -d & -d^{*} & d^{*} d+c^{*} c \\
b d^{*}-d c^{*} & b & c^{*} & c^{*} d-d^{*} b \\
d b^{*}-c d^{*} & c & b^{*} & d^{*} c-b^{*} d \\
c c^{*}+d d^{*} & d & d^{*} & b^{*} b+d^{*} d
\end{array}\right]
$$


describing the action of $\boldsymbol{G}$ on elements of the basis $\left\{\boldsymbol{n} \boldsymbol{n}^{*}, \boldsymbol{n}, \boldsymbol{n}^{*}, \boldsymbol{n}^{*} \boldsymbol{n}\right\}$ is unitary. Now the same reasoning as presented in the proof of Theorem 4.3 shows that $c$ is normal.

Using the normality of $c$ and relations (51) we easily show that $b$ and $d$ are also normal. Then, using Theorem 1.2 we show that $b, c, d$ and their adjoints all commute. Moreover $b c=-d^{2}$ and

$$
2|d|^{2}+|b|^{2}+|c|^{2}=\mathbb{1} .
$$

Let us define the following subset of $\mathbb{C}^{3}$ :

$$
\mathbb{S}=\left\{\left[\begin{array}{l}
s \\
t \\
r
\end{array}\right] \in \mathbb{C}^{3}\left|s t=-r^{2},\right| s|+| t \mid=1\right\} .
$$

It is an amusing exercise to show that $\$$ is homeomorphic to $\mathbb{R P}(3)$ and the multiplication

$$
\left[\begin{array}{l}
s \\
t \\
r
\end{array}\right] \cdot\left[\begin{array}{c}
s^{\prime} \\
t^{\prime} \\
r^{\prime}
\end{array}\right]=\left[\begin{array}{c}
2(r \bar{t}-s \bar{r}) r^{\prime}+s s^{\prime}+\bar{t} t^{\prime} \\
2(t \bar{r}-r \bar{s}) r^{\prime}+t s^{\prime}+\bar{s} t^{\prime} \\
\left(|s|^{2}-|t|^{2}\right) r^{\prime}+r s^{\prime}+\bar{r} t^{\prime}
\end{array}\right]
$$

gives this space a locally compact group structure with which $\mathbb{\$}$ is isomorphic to $\mathrm{SO}(3)$. The unit element is

$$
\left[\begin{array}{l}
1 \\
0 \\
0
\end{array}\right]
$$

and the inverse is described by

$$
\left[\begin{array}{l}
s \\
t \\
r
\end{array}\right]^{-1}=\left[\begin{array}{l}
\bar{s} \\
t \\
x
\end{array}\right]
$$

where

$$
x= \begin{cases}\frac{|s| t}{r} & r \neq 0, \\ 0 & \text { otherwise. }\end{cases}
$$

Using the above description of $\mathrm{SO}(3)$ we can view $\mathrm{C}(\mathrm{SO}(3))$ and its standard comultiplication in the following way: $\mathrm{C}(\mathrm{SO}(3))$ is the universal $\mathrm{C}^{*}$-algebra generated by three normal and commuting elements $S, T$ and $R$ satisfying the relations

$$
S T=-R^{2}, \quad|S|+|T|=\mathbb{1} .
$$

The comultiplication acts on generators in the following way

$$
\begin{aligned}
& S \longmapsto 2\left(R T^{*}-S R^{*}\right) \otimes R+S \otimes S+T^{*} \otimes T, \\
& T \longmapsto 2\left(T R^{*}-R S^{*}\right) \otimes R+T \otimes S+S^{*} \otimes T, \\
& R \longmapsto\left(S^{*} S-T^{*} T\right) \otimes R+R \otimes S+R^{*} \otimes T .
\end{aligned}
$$


Now we see that there exists a unique $\Gamma \in \operatorname{Mor}(\mathrm{C}(\operatorname{SO}(3)), \mathfrak{B})$ such that

$$
\Gamma(S)=b, \quad \Gamma(T)=c, \quad \Gamma(R)=d .
$$

One can check that this map intertwines the standard action of $\mathrm{SO}(3)$ on $M_{2}$ with $\Psi_{\mathfrak{B}}$. Moreover this condition determines $\Gamma$ uniquely. We have thus proved the following result.

Theorem 5.2. Let $\mathcal{E}=\left(\mathfrak{B}, \Delta_{\mathfrak{B}}\right)$ be a compact quantum group and let $\Psi_{\mathfrak{B}} \in$ $\operatorname{Mor}\left(M_{2}, M_{2} \otimes \mathfrak{B}\right)$ be a continuous action of $\mathscr{G}$ on $M_{2}$ preserving the trace. Then there exists a unique $\Gamma \in \operatorname{Mor}(\mathrm{C}(\mathrm{SO}(3)), \mathfrak{B})$ such that

$$
\left(\operatorname{id}_{M_{2}} \otimes \Gamma\right) \circ \Psi_{1}=\Psi_{\mathfrak{B}},
$$

where $\Psi_{1} \in \operatorname{Mor}\left(M_{2}, M_{2} \otimes \mathrm{C}(\mathrm{SO}(3))\right)$ is the morphism describing the standard action of $\mathrm{SO}(3)$ on $M_{2}$. Moreover $\Gamma$ is a compact quantum group morphism.

5.2. Case $\boldsymbol{q}=\mathbf{0}$. Since there is no obvious definition of $\mathrm{S}_{0} \mathrm{O}(3)$, let us first describe the compact quantum semigroup $Q-\operatorname{Map}^{\omega_{q}}\left(\mathcal{Q S}\left(M_{2}\right)\right)$ for $q=0$. Note that in this case the state $\omega_{q}$ is not faithful. We can easily find the $\mathrm{C}^{*}$-algebra with comultiplication $(\boldsymbol{A}, \boldsymbol{\Delta})$ describing this quantum semigroup.

Proposition 5.3. For $q=0$ the $C^{*}$-algebra $\boldsymbol{A}$ is the universal $C^{*}$-algebra generated by two elements $\beta$ and $\delta$ satisfying the relations

$$
\begin{aligned}
\beta \beta^{*} & =\mathbb{1}, & \delta^{2}=0, \\
\beta \delta & =0, & \beta \delta^{*}=0, \\
\beta^{*} \beta+\delta^{*} \delta+\delta \delta^{*} & =\mathbb{1} . &
\end{aligned}
$$

The comultiplication $\boldsymbol{\Delta}$ acts on generators in the following way:

$$
\begin{aligned}
& \Delta(\beta)=\beta \otimes \beta, \\
& \boldsymbol{\Delta}(\delta)=\delta \otimes \beta+\beta^{*} \beta \otimes \delta+\delta^{*} \delta \otimes \delta,
\end{aligned}
$$

while the counit maps $\beta$ to 1 and $\delta$ to 0 .

The action of $\mathrm{Q}-\mathrm{Map}^{\omega_{q}}\left(\mathrm{QS}\left(M_{2}\right)\right)$ on $\mathcal{Q S}\left(M_{2}\right)$ is given by $\Phi \in \operatorname{Mor}\left(M_{2}, M_{2} \otimes A\right)$ defined by

$$
\boldsymbol{\Phi}(\boldsymbol{n})=\left[\begin{array}{ll}
0 & \beta \\
0 & \delta
\end{array}\right] .
$$

The proof of Proposition 5.3 is completely analogous to that of Proposition 2.2.

Remark 5.4. Let us note that the relations (52) can be realized in the following way: let $H$ be an infinite dimensional Hilbert space and let $U$ be a unitary map 
$H \rightarrow H \oplus H \oplus H$. Then we can put

$$
\begin{aligned}
& \beta: H \oplus H \oplus H \ni\left[\begin{array}{l}
x \\
y \\
z
\end{array}\right] \longmapsto U x \in H \oplus H \oplus H, \\
& \delta: H \oplus H \oplus H \ni\left[\begin{array}{l}
x \\
y \\
z
\end{array}\right] \longmapsto\left[\begin{array}{l}
0 \\
0 \\
y
\end{array}\right] \in H \oplus H \oplus H .
\end{aligned}
$$

Note that in this case $\delta \beta \neq 0=\beta \delta, \delta^{*} \beta \neq 0=\beta \delta^{*}$ and $\beta^{*} \beta \neq \mathbb{1}=\beta \beta^{*}$. In particular $\delta \neq 0$ in $\boldsymbol{A}$ and $\boldsymbol{A}$ is not commutative. In fact it clearly contains a copy of the Toeplitz algebra.

Proposition 5.5. The quantum semigroup $Q-\operatorname{Map}^{\omega_{0}}\left(Q \mathcal{S}\left(M_{2}\right)\right)$ is not a compact quantum group.

Proof. Assume that $(\boldsymbol{A}, \boldsymbol{\Delta})$ is a compact quantum group. The element $Y=\beta^{*} \beta$ is a group-like projection. If the Haar measure of $(\boldsymbol{A}, \boldsymbol{\Delta})$ were faithful, $Y$ would have to belong to the dense Hopf $*$-algebra $\mathcal{A}$ of $\boldsymbol{A}$ (by [21], Theorem 2.6(2)). In that case the antipode of $Y$ would have to be its inverse. However, we know that in $\boldsymbol{A}$ the element $Y$ is a proper projection (cf. Remark 5.4), and as such cannot be invertible.

This means that the Haar measure of $(\boldsymbol{A}, \boldsymbol{\Delta})$ is not faithful and the image of $Y$ under the reducing map $\lambda: \boldsymbol{A} \rightarrow \boldsymbol{A}_{r}$ ([19], p. 656) is either $\mathbb{1}$ or 0 .

If $\lambda(Y)=\mathbb{1}$ then $\lambda(\delta)$ must be 0 and $\boldsymbol{A}_{r}$ is generated by a single unitary $\lambda(\beta)$. In that case, however, the $\mathrm{C}^{*}$-algebra $\boldsymbol{A}_{r}$ is commutative and thus the quantum group $\left(\boldsymbol{A}_{r}, \boldsymbol{\Delta}_{r}\right)$ is not only reduced, but also universal. Therefore we must have $\delta=0$ in $\boldsymbol{A}$ which is not true by Remark 5.4.

The only remaining possibility is that $\lambda(Y)=0$. In this case $\lambda(\beta)=0$ and $\boldsymbol{A}_{r}$ is generated by $x=\lambda(\delta)$ which satisfies

$$
x^{2}=0 \text { and } \quad x x^{*}+x^{*} x=\mathbb{1} .
$$

It follows that $\boldsymbol{A}_{r}=M_{2}$. However this $\mathrm{C}^{*}$-algebra does not admit a compact quantum group structure. This contradiction shows that the assumption that $(\boldsymbol{A}, \boldsymbol{\Delta})$ was a compact quantum group is false.

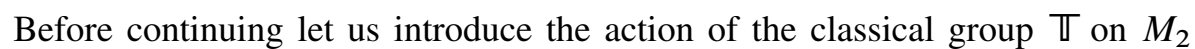
preserving $\omega_{0}$. Let $\boldsymbol{u}$ be the standard generator of $\mathrm{C}(\mathbb{\mathbb { T }})$ then the morphism $\Psi_{0} \in$ $\operatorname{Mor}\left(M_{2}, M_{2} \otimes \mathrm{C}(\mathbb{T})\right)$,

$$
\Psi_{0}(\boldsymbol{n})=\left[\begin{array}{ll}
0 & \boldsymbol{u} \\
0 & 0
\end{array}\right],
$$

describes the action by automorphism sending for each $\mathrm{e}^{\mathrm{i} \varphi} \in \mathbb{T}$ the element $\boldsymbol{n}$ to $\mathrm{e}^{\mathrm{i} \varphi} \boldsymbol{n}$. Now a very similar reasoning to that given in the proof of Proposition 5.5 leads to the following result.

Theorem 5.6. Let $\mathcal{E}=\left(\mathfrak{B}, \Delta_{\mathfrak{B}}\right)$ be a compact quantum group and let $\Psi_{\mathfrak{B}} \in$ $\operatorname{Mor}\left(M_{2}, M_{2} \otimes \mathfrak{B}\right)$ be a continuous action of $\mathcal{G}$ on $\mathcal{Q S}\left(M_{2}\right)$ preserving the state 
$\omega_{0}$. Then there exists a unique $\Gamma \in \operatorname{Mor}(\mathrm{C}(\mathbb{T}), \mathfrak{B})$ such that

$$
\left(\operatorname{id}_{M_{2}} \otimes \Gamma\right) \circ \Psi_{0}=\Psi_{\mathfrak{B}} .
$$

Proof. We keep the notation from Proposition 5.3. Let $\Lambda \in \operatorname{Mor}(\boldsymbol{A}, \mathfrak{B})$ be the unique morphism satisfying

$$
\left(\mathrm{id}_{M_{2}} \otimes \Lambda\right) \circ \boldsymbol{\Phi}=\Psi_{\mathfrak{B}}
$$

and let

$$
b=\Lambda(\beta), \quad d=\Lambda(\delta) .
$$

In terms of $b$ and $d$ the action of $\mathscr{G}$ on $\mathcal{Q S}\left(M_{2}\right)$ is given by

$$
\Psi_{\mathfrak{B}}(\boldsymbol{n})=\left[\begin{array}{ll}
0 & b \\
0 & d
\end{array}\right] .
$$

We know that $b$ and $d$ belong to the dense Hopf $*$-subalgebra $\mathscr{B}$ of $\mathfrak{B}$. Moreover

$$
\begin{aligned}
& \Delta_{\mathfrak{B}}(b)=b \otimes b, \\
& \Delta_{\mathfrak{B}}(d)=d \otimes b+b^{*} b \otimes d+d^{*} d \otimes d .
\end{aligned}
$$

As noted in the proof of Proposition 5.5, $b^{*} b$ is a group-like projection in $\mathscr{B}$, and so it is either 0 of or $\mathbb{1}$.

The possibility $b=0$ can be excluded in many ways. Either we show that in that case $d^{*} d$ is a proper projection which is also group-like or we use the fact that $d^{2}=0$ implies that $\epsilon_{\mathscr{B}}(d)=0$, so that $d^{*} d=\left(\operatorname{id}_{\mathscr{B}} \otimes \epsilon_{\mathscr{B}}\right)\left(d^{*} d \otimes d\right)=0$, which is impossible.

This means that $b$ is unitary and $d=0$. Consequently there exists a unique $\Gamma \in \operatorname{Mor}(\mathrm{C}(\mathbb{Z}), \mathfrak{B})$ such that

$$
\Gamma(\boldsymbol{u})=b .
$$

Comparing (53) and (55) shows that (56) is equivalent to (54).

\section{General actions on $M_{2}$}

Let $\mathcal{E}=\left(\mathfrak{B}, \Delta_{\mathfrak{B}}\right)$ be a compact quantum group acting continuously on $\mathcal{Q S}\left(M_{2}\right)$. Let $\Psi_{\mathfrak{B}} \in \operatorname{Mor}\left(M_{2}, M_{2} \otimes \mathfrak{B}\right)$ be the morphism describing this action. Then there exists an invariant state for $\Psi_{\mathfrak{B}}$. Indeed let $h$ be that Haar measure of $\mathscr{E}$ and $\phi$ be any state on $M_{2}$. Then $\eta=(\phi \otimes h) \circ \Psi_{\mathfrak{B}}$ is an invariant state: for any $\in M_{2}$ and $\mu \in \mathfrak{B}^{\prime}$ we have (cf. [3], Lemma 4)

$$
\begin{aligned}
\mu\left(\left(\eta \otimes \operatorname{id}_{\mathfrak{B}}\right) \Psi_{\mathfrak{B}}(m)\right) & =\mu\left(\left(\phi \otimes h \otimes \operatorname{id}_{\mathfrak{B}}\right)\left(\Psi_{\mathfrak{B}} \otimes \operatorname{id}_{\mathfrak{B}}\right) \Psi_{\mathfrak{B}}(m)\right) \\
& =(\phi \otimes h \otimes \mu)\left(\operatorname{id}_{M_{2}} \otimes \Delta_{\mathfrak{B}}\right) \Psi_{\mathfrak{B}}(m) \\
& =\left(\phi \otimes \operatorname{id}_{\mathfrak{B}}\right)(\operatorname{id} \otimes[h * \mu]) \Psi_{\mathfrak{B}}(m) \\
& =\left(\phi \otimes \operatorname{id}_{\mathfrak{B}}\right)\left(\operatorname{id}_{M_{2}} \otimes h\right) \Psi_{\mathfrak{B}}(m) \mu(\mathbb{1})=\mu(\eta(m) \mathbb{1}) .
\end{aligned}
$$


States on $M_{2}$ correspond to density matrices, so for $\eta$ there exists a unique $\rho$ such that $\eta(m)=\operatorname{tr}(\rho m)$ for all $m$ and which is conjugate to one of the matrices

$$
\rho_{q}=\frac{1}{1+q^{2}}\left[\begin{array}{cc}
1 & 0 \\
0 & q^{2}
\end{array}\right] \quad(q \in[0,1]) .
$$

Note also that $\operatorname{tr}\left(\rho_{q} m\right)=\omega_{q}(m)$. Let $u$ be the unitary element of $M_{2}$ such that $\rho=u \rho_{q} u^{*}$. Then $\eta\left(u m u^{*}\right)=\omega_{q}(m)$ for all $m$. Therefore if we define

$$
\widetilde{\Psi}_{\mathfrak{B}}: M_{2} \ni m \longmapsto\left(u^{*} \otimes \mathbb{1}\right) \Psi_{\mathfrak{B}}\left(u m u^{*}\right)\left(u^{*} \otimes \mathbb{1}\right) \in M_{2} \otimes \mathfrak{B}
$$

we obtain an action $\widetilde{\Psi}_{\mathfrak{B}} \in \operatorname{Mor}\left(M_{2}, M_{2} \otimes \mathfrak{B}\right)$ : for all $m \in M_{2}$ we have

$$
\begin{aligned}
\left(\widetilde{\Psi}_{\mathfrak{B}} \otimes \operatorname{id}_{\mathfrak{B}}\right) \widetilde{\Psi}_{\mathfrak{B}}(m)= & \left(u^{*} \otimes \mathbb{1} \otimes \mathbb{1}\right)\left[\left(\Psi_{\mathfrak{B}} \otimes \operatorname{id}_{\mathfrak{B}}\right)\left((u \otimes \mathbb{1})\left(\widetilde{\Psi}_{\mathfrak{B}}(m)\right)\left(u^{*} \otimes \mathbb{1}\right)\right)\right] \\
& \cdot(u \otimes \mathbb{1} \otimes \mathbb{1}) \\
= & \left(u^{*} \otimes \mathbb{1} \otimes \mathbb{1}\right)\left[\left(\Psi_{\mathfrak{B}} \otimes \operatorname{id}_{\mathfrak{B}}\right) \Psi_{\mathfrak{B}}\left(u m u^{*}\right)\right](u \otimes \mathbb{1} \otimes \mathbb{1}) \\
= & \left(u^{*} \otimes \mathbb{1} \otimes \mathbb{1}\right)\left[\left(\operatorname{id}_{M_{2}} \otimes \Delta_{\mathfrak{B}}\right) \Psi_{\mathfrak{B}}\left(u m u^{*}\right)\right](u \otimes \mathbb{1} \otimes \mathbb{1}) \\
= & \left(\operatorname{id}_{M_{2}} \otimes \Delta_{\mathfrak{B}}\right)\left(\left(u^{*} \otimes \mathbb{1}\right) \Psi_{\mathfrak{B}}\left(u m u^{*}\right)(u \otimes \mathbb{1})\right) \\
= & \left(\operatorname{id}_{M_{2}} \otimes \Delta_{\mathfrak{B}}\right) \widetilde{\Psi}_{\mathfrak{B}}(m),
\end{aligned}
$$

which is continuous because

$$
\begin{aligned}
\left\{\widetilde{\Psi}_{\mathfrak{B}}(m)\right. & \left.(\mathbb{1} \otimes b) \mid m \in M_{2}, b \in \mathfrak{B}\right\} \\
& =\left\{\left(u^{*} \otimes \mathbb{1}\right) \Psi_{\mathfrak{B}}\left(u m u^{*}\right)(u \otimes \mathbb{1})\left(\mathbb{1} \otimes b \mid m \in M_{2}, b \in \mathfrak{B}\right\}\right. \\
& =\left(u^{*} \otimes \mathbb{1}\right)\left\{\Psi_{\mathfrak{B}}(k)\left(\mathbb{1} \otimes b \mid k \in M_{2}, b \in \mathfrak{B}\right\}(u \otimes \mathbb{1})\right.
\end{aligned}
$$

is linearly dense in $M_{2} \otimes \mathfrak{B}$. Moreover for any $m \in M_{2}$ we have

$$
\begin{aligned}
\left(\omega_{q} \otimes \operatorname{id}_{\mathfrak{B}}\right) \widetilde{\Psi}_{\mathfrak{B}}(m) & =\left(\omega_{q} \otimes \operatorname{id}_{\mathfrak{B}}\right)\left(\left(u^{*} \otimes \mathbb{1}\right) \Psi_{\mathfrak{B}}\left(u m u^{*}\right)(u \otimes \mathbb{1})\right) \\
& =\left(\eta \otimes \operatorname{id}_{\mathfrak{B}}\right) \widetilde{\Psi}_{\mathfrak{B}}\left(u m u^{*}\right) \\
& =\eta\left(u m u^{*}\right) \mathbb{1}=\omega_{q}(m) \mathbb{\mathbb { 1 }},
\end{aligned}
$$

so $\widetilde{\Psi}$ preserves $\omega_{q}$.

In order to state the next theorem in a readable way let us use the symbol $\mathrm{S}_{0} \mathrm{O}(3)$ for the group $\mathbb{T}$. For each $q \in[0,1]$ the symbol $\Psi_{q}$ denotes the action of $\mathrm{S}_{q} \mathrm{O}(3)$ on $Q \mathcal{S}\left(M_{2}\right)$ described in Sections 3.2, 5.1 and 5.2.

Applying the results of Sections 4 and 5 we get the following description of all continuous actions of compact quantum groups on $M_{2}$.

Theorem 6.1. Let $\mathcal{E}=\left(\mathfrak{B}, \Delta_{\mathfrak{B}}\right)$ be a compact quantum group and let $\Psi_{\mathfrak{B}} \in$ $\operatorname{Mor}\left(M_{2}, M_{2} \otimes \mathfrak{B}\right)$ be a continuous action of $\mathscr{E}$ on $Q \mathcal{S}\left(M_{2}\right)$. Then there exists a unitary $u \in M_{2}, q \in[0,1]$ and $\Gamma \in \operatorname{Mor}\left(\mathrm{C}\left(\mathrm{S}_{q} \mathrm{O}(3)\right)\right.$, $\left.\mathfrak{B}\right)$ such that

$$
\Psi_{\mathfrak{B}}(m)=\left(\operatorname{id}_{M_{2}} \otimes \Gamma\right)\left((u \otimes \mathbb{1}) \Psi_{q}\left(u^{*} m u\right)\left(u^{*} \otimes \mathbb{1}\right)\right) .
$$

$\Gamma$ and $u$ are unique for each $q$. Moreover, if $\Psi_{\mathfrak{B}}$ is ergodic then $q$ is unique. 
The only element of Theorem 6.1 that requires a comment at this stage is the uniqueness statement. It follows from the fact that for an ergodic continuous action of a compact quantum group there exists a unique invariant state ([3], Lemma 4) whose density matrix is then conjugate to a unique matrix from the family (57).

\section{References}

[1] J. Bhowmick and D. Goswami, Quantum isometry groups of the Podleś spheres. Preprint 2009. arXiv:0810.0658

[2] J. Bhowmick and D. Goswami, Quantum isometry groups: examples and computations. Comm. Math. Phys. 285 (2009), 421-444. Zbl 1159.81028 MR 2461983

[3] F. P. Boca, Ergodic actions of compact matrix pseudogroups on $C *$-algebras. Astérisque 232 (1995), 93-109. Zbl 0842.46039 MR 1372527

[4] L. Dạbrowski, F. D'Andrea, G. Landi, and E. Wagner, Dirac operators on all Podleś quantum spheres. J. Noncommut. Geom. 1 (2007), 213-239. Zbl 1121.58008 MR 2308305

[5] D. Goswami, Quantum group of isometries in classical and noncommutative geometry. Comm. Math. Phys. 285 (2009), 141-160. MR 2453592

[6] M. Izumi, Non-commutative Poisson boundaries and compact quantum group actions. Adv. Math. 169 (2002), 1-57. Zbl 1037.46056 MR 1916370

[7] M. Marciniak, Quantum symmetries in noncommutative $C$ *-systems. In Quantum probability (Gdańsk, 1997), Banach Center Publ. 43, Polish Acad. Sci., Warsaw 1998, 297-307. Zbl 0927.46051 MR 1649732

[8] P. Podleś, Quantum spheres. Lett. Math. Phys. 14 (1987), 193-202. Zbl 0634.46054 MR 0919322

[9] P. Podleś, Przestrzenie kwantowe i ich grupy symetrii (Quantum spaces and their symmetry groups). PhD Thesis, Department of Mathematical Methods in Physics, Warsaw University, Warsaw 1989.

[10] P. Podleś, Symmetries of quantum spaces. Subgroups and quotient spaces of quantum $S U(2)$ and SO(3) groups. Comm. Math. Phys. 170 (1995), 1-20. Zbl 0853.46074 MR 1331688

[11] P. Podleś and E. Müller, Introduction to quantum groups. Rev. Math. Phys. 10 (1998), 511-551. Zbl 0918.17005 MR 1629723

[12] W. Rudin, Functional analysis. McGraw-Hill Book Co., New York 1973. Zbl 0253.46001 MR 0365062

[13] P. M. Sołtan, Examples of quantum commutants. Arab. J. Sci. Eng. 33 (2008), no. 2C, 447-457. MR 2500052 http://ajse.kfupm.edu.sa/articles/332C_P.29.pdf

[14] P. M. Sołtan, Quantum families of maps and quantum semigroups on finite quantum spaces. J. Geom. Phys. 59 (2009), 354-368. Zbl 1160.58007 MR 2501746

[15] S. Vaes, A new approach to induction and imprimitivity results. J. Funct. Anal. 229 (2005), 317-374. Zbl 1087.22005 MR 2182592 
[16] S. Wang, Quantum symmetry groups of finite spaces. Comm. Math. Phys. 195 (1998), 195-211. Zbl 1013.17008 MR 1637425

[17] S. L. Woronowicz, Pseudospaces, pseudogroups and Pontrjagin duality. In Mathematical problems in theoretical physics (Proc. Internat. Conf. Math. Phys., Lausanne, 1979), Lecture Notes in Phys. 116, Springer, Berlin 1980, 407-412. Zbl 0513.46046 MR 0582650

[18] S. L. Woronowicz, Twisted SU(2) group. An example of a noncommutative differential calculus. Publ. Res. Inst. Math. Sci. 23 (1987), 117-181. Zbl 0676.46050 MR 890482

[19] S. L. Woronowicz, Compact matrix pseudogroups. Comm. Math. Phys. 111 (1987), 613-665. Zbl 0627.58034 MR 901157

[20] S. L. Woronowicz, Unbounded elements affiliated with $C^{*}$-algebras and non-compact quantum groups. Commun. Math. Phys. 136 (1991), 399-432. Zbl 0743.46080 MR 1096123

[21] S. L. Woronowicz, Compact quantum groups. In Symétries quantiques (Les Houches, 1995), North-Holland, Amsterdam 1998, 845-884. Zbl 0997.46045 MR 1616348

[22] S. L. Woronowicz, Quantum exponential function. Rev. Math. Phys. 12 (2000), 873-920. Zbl 0961.47013 MR 1770545

Received October 6, 2008

P. M. Sołtan, Institute of Mathematics of the Polish Academy of Sciences, ul. Śniadeckich 8, P.O. Box 21, 00-956 Warszawa

Department of Mathematical Methods in Physics, Faculty of Physics, University of Warsaw, Hoza 74, 00-682 Warsaw, Poland

E-mail: piotr.soltan@fuw.edu.pl 